\title{
Sur les Classes Caractéristiques des Sous-Feuilletages
}

\author{
par \\ Demetrio Domínguez*
}

\begin{abstract}
The purpose of this paper is twofold. First, we compute the cohomology algebras $H$ ( W( $g$. $\left.H)_{I}\right)$; and second, we study some properties of subfoliations, in order to give a geometric interpretation for certain secondary characteristic classes of subfoliations, in particular, Godbillon-Vey's classes of a subfoliation. We also construct examples of subfoliations with non-trivial Godbillon-Vey classes.
\end{abstract}

\section{$\S 1$. Introduction}

Soit $M$ une variété différentiable de dimension $n$, TM son fibré tangent. Un sous-feuilletage de codimension $\left(q_{1}, q_{2}\right)$ sur $M$ est une paire $\left(F_{1}\right.$, $F_{2}$ ) de sous-fibrés intégrables $F_{2}$ de $T M$ de dimensions $n-q_{2}, i=1$, 2 , et tels que $F_{2}$ soit un sous-fibré de $F_{1}$. Les classes caractéristiques secondaires ou exotiques ont été définies pour les sous-feuilletages de codimension $\left(q_{1}, q_{2}\right)$, par Cordero-Masa [3], en utilisant les techniques de Bott [1]. Finalement, Carballés [2], en utilisant les techniques de Kamber-Tondeur [8], généralise la construction de Cordero-Masa en introduisant l'homomorphisme caractéristique $\Delta_{*}=\Delta_{*}(P): H\left(W(g, H)_{I}\right) \longrightarrow H_{D R}(M)$ d'un fibré principal $\left(F_{1}, F_{2}\right)$-feuilleté $P \longrightarrow M$. C'est un fibré principal de la forme $P=P_{1}+P_{2}$ de groupe structural $G=G_{1} \times G_{2}$ tel que $P_{2} \longrightarrow M, i=1$, 2, soit un fibré principal $F_{2}$-feuilleté de groupe structural $G_{2}$.

Le but de ce travail est double : d'une part calculer la cohomologie de l'algèbre de Weil relative tronquée $W(g, h)_{I}$ pour certaines paires

Communiqué par N. Shimada, le 11 mai 1987.

* Departamento de Geometría y Topología, Facultad de Matemáticas, Universidad de Santiago de Compostela, Santiago de Compostela, Spain. 
réductives $(g, h)=\left(g_{1} \oplus g_{2}, h_{1} \oplus h_{2}\right)$ d'algèbres de Lie, d'autre part étudier quelques propriétés des sous-feuilletages, en interprétant géométriquement certaines classes caractéristiques secondaires, en particulier, les nouvelles classes de Godbillon-Vey d'un sous-feuilletage.

Le $\S 2$ est consacré à quelques rappels concernant en particulier l'homomorphisme caractéristique généralisé $\Delta_{*}$ d'un fibré principal $\left(F_{1}\right.$, $F_{2}$ )-feuilleté $P=P_{1}+P_{2}$. D'autre part, nous montrons que l'homomorphisme caractéristique généralisé $\Delta_{*}$ d'un fibré principal $\left(F_{1}, F_{2}\right)$-feuilleté $P$ (admettant une connexion basique somme $\omega=\omega_{1}+\omega_{2}$, au sens de Carballés [2]) ne dépend pas du choix de la connexion basique somme sur $P$.

Le $\S 3$ contient le calcul de la cohomologie de l'algèbre de Weil relative tronquée $W(g, h)_{I}$ pour certaines paires réductives $(g, h)=\left(g_{1} \oplus g_{2}, h_{1} \oplus h_{2}\right)$ d'algèbres de Lie de dimension finie sur un corps commutatif $\Re$ de caractéristique zéro. Ceci nous permet d'introduire la notion de classes de Godbillon-Vey pour les sous-feuilletages de codimension $\left(q_{1}, q_{2}\right)$.

Le $\S 4$ étudie la compatibilité des structures $F_{2}$-feuilletées canoniques des fibrés vectoriels isomorphes $Q_{2}=T M / F_{2}$ et $Q_{1} \oplus Q_{0}=\left(T M / F_{1}\right) \oplus\left(F_{1} / F_{2}\right)$. On interprète en particulier les classes de Godbillon-Vey d'un sousfeuilletage $\left(F_{1}, F_{2}\right)$ de codimension $\left(q_{1}, q_{2}\right)$. Ce paragraphe contient aussi le cas où le sous-feuilletage $\left(F_{1}, F_{2}\right)$ est de la forme $\left(F_{1}, F_{1} \cap F^{\prime}\right)$ pour un feuilletage $F^{\prime}$ de codimension $d=q_{2}-q_{1} \geqq 0$ sur $M$.

Le $\S 5$ montre que les classes de Godbillon-Vey d'un sous-feuilletage peuvent s'obtenir en utilisant des techniques analogues à celles utilisées par Godbillon-Vey [5] pour un feuilletage.

$\mathrm{Au} \S 6$ nous donnons quelques exemples de sous-feuilletages localement homogènes pour appliquer les résultats des paragraphes précédents. En particulier, nous calculons les classes de Godbillon-Vey de ces sousfeuilletages.

Tout au long de ce travail, toutes les variétés et toutes les applications seront différentiables de classe $C^{\infty}$. Nous adoptons aussi les notations de Kamber-Tondeur [8] et Carballés [2]. De même, on désignera par $g l(m)$ (resp. par $s l(m)$ ) l'algèbre de Lie du groupe $G L(m)=G L(m, \Re)$ (resp. du groupe $S L(m)=S L(m, \Re)$ ).

Ce travail est une partie de la dissertation doctoral de l'auteur qui exprime sa très grande reconnaissance à X. M. Masa pour son aide et encouragement. 


\section{§ 2. Homomorphisme Caractéristique d'un Fibrê $\left(F_{1}, F_{2}\right)$-Feuilletê}

Soient $H \subset G=G_{1} \times G_{2}$ des groupes de Lie, $h \subset g=g_{1} \oplus g_{2}$ leurs algèbres de Lie. Pour une paire $\left(q_{1}, q_{2}\right)$ d'entiers $q_{2}$ tels que $0 \leqq q_{1} \leqq q_{2}$, on désignera par $W(g, H)_{I}$ l'algèbre de Weil relative tronquée $\left(W(g)_{I}\right)_{H}$ des éléments $H$ basiques de l'algèbre de Weil $W(g)_{I}=W(g) / I$ tronquée par l'idéal $I$ $=^{\prime} F^{2\left(q_{1}+1\right)} W(g)+F^{2\left(q_{2}+1\right)} W(g)$ de l'algèbre de Weil $W(g) \cong W\left(g_{1}\right) \otimes W\left(g_{2}\right)$ engendré par les sous-espaces $S^{\imath} g_{1}{ }^{*} \otimes S^{\jmath} g_{2}{ }^{*}$ tels que $i>q_{1}$ ou $i+j>q_{2}$.

Soit $\left(F_{1}, F_{2}\right)$ un sous-feuilletage de codimension $\left(q_{1}, q_{2}\right)$ sur une variété différentiable $M$ de dimension $n$ et soit $P=P_{1}+P_{2} \longrightarrow M$ un fibré principal $\left(F_{1}, F_{2}\right)$-feuilleté de groupe structural $G=G_{1} \times G_{2}$. Supposons que $H$ soit fermé dans $G$ et qu'il existe une section $s: M \longrightarrow P / H$ de la projection canonique $\hat{\pi}: P / H \longrightarrow M$. Alors, d'après Carballés [2], l'homomorphisme caractéristique généralisé

$$
\Delta_{*}=\Delta_{*}(P): H\left(W(g, H)_{I}\right) \longrightarrow H_{D R}(M)
$$

du fibré principal $\left(F_{1}, F_{2}\right)$-feuilleté $P$ est l'homomorphisme induit en cohomologie par le DG-homomorphisme composé

$$
\Delta(\omega)=s^{*} \circ k(\omega)_{H}: W(g, H)_{I} \longrightarrow \Omega(P)_{H} \cong \Omega(P / H) \longrightarrow \Omega(M),
$$

où $k(\omega): W(g) \longrightarrow \Omega(P)$ désigne l'homomorphisme de Weil d'une connexion adaptée somme $\omega=\omega_{1}+\omega_{2}$ sur $P=P_{1}+P_{2}$ (au sens de Carballés [2]). De plus, l'homomorphisme $\Delta_{*}$ ne dépend pas du choix de la connexion adaptée somme $\omega$ sur $P$. De même, si $H$ contient un sous-groupe maximal compact de $G$, l'homomorphisme $\Delta_{*}$ est aussi indépendant de la section $s$ choisie.

Considérons maintenant le fibré principal $\left(F_{1}, F_{2}\right)$-feuilleté $P=L\left(Q_{1}\right)$ $+L\left(Q_{0}\right)$ des repères transverses au sous-feuilletage $\left(F_{1}, F_{2}\right)$, c'est-à-dire le fibré des $G L\left(q_{1}\right) \times G L(d)$-repères du fibré normal $\nu\left(F_{1}, F_{2}\right)=Q_{1} \oplus Q_{0}$ de $\left(F_{1}\right.$, $F_{2}$ ), où $d=q_{2}-q_{1} \geqq 0$. Alors (cf. Carballés [2]), pour $H=O\left(q_{1}\right) \times O(d)$ (ou $H=\{e\}$, dans le cas où le fibré normal de ce sous-feuilletage est trivialisé), l'homomorphisme $\Delta_{*}=\Delta_{*}(P)$ coïncide avec l'homomorphisme caractéristique $\lambda_{\left(F_{1}, F_{2}\right)}^{*}$ défini par Cordero-Masa [3]. Dans ce cas, les éléments de Im $\Delta_{*}$ $\subset H_{D R}(M)$ seront appelés des classes caractéristiques de $\left(F_{1}, F_{2}\right)$.

D'autre part, supposons que le fibré principal $\left(F_{1}, F_{2}\right)$-feuilleté $P=P_{1}$ $+P_{2} \longrightarrow M$ admette une connexion basique somme $\omega=\omega_{1}+\omega_{2}$ (au sens de 
Carballés [2]), alors, par un raisonnement analogue à celui utilisé dans [2], nous obtenons le résultat suivant.

Proposition 2.1. L'homomorphisme caractéristique généralisé

$$
\Delta_{*}: H\left(W(g, H)_{I^{\prime \prime}}\right) \longrightarrow H_{D R}(M)
$$

du fibré principal $\left(F_{1}, F_{2}\right)$-feuilleté $P=P_{1}+P_{2}$ ne dépend pas du choix de la connexion basique somme $\omega=\omega_{1}+\omega_{2}$ sur $P$, où $I^{\prime \prime}$ est le $G$-DG-idéal de l'algèbre de Weil $W(g) \cong W\left(g_{1}\right) \otimes W\left(g_{2}\right)$ correspondant à la paire $\left(\left[\left(q_{1}+1\right) /\right.\right.$ 2], $\left.\left[\left(q_{2}+1\right) / 2\right]\right)$.

Soit la situation précédente. Alors l'homomorphisme $\Delta_{*}$ se factorise de la manière suivante:

$$
\Delta_{*}: H\left(W(g, H)_{I^{\prime \prime}}\right) \stackrel{p_{*}}{\longrightarrow} H\left(W(g, H)_{I^{\prime}}\right) \longrightarrow H_{D R}(M),
$$

où $p$ est la projection canonique induite par l'inclusion

$$
I^{\prime \prime} \subset I^{\prime}=F^{2\left(\left[q_{1} / 2\right]+1\right)} W(g)+F^{2\left(\left[q_{2} / 2\right]+1\right)} W(g),
$$

mais l'homomorphisme $\Delta_{*}=\Delta(\omega)_{*}: H\left(W(g, H)_{I^{\prime}}\right) \longrightarrow H_{D R}(M)$ dépend, en général, du choix de la connexion basique somme $\omega=\omega_{1}+\omega_{2}$ sur $P=P_{1}+P_{2}$ si $q_{1}$ ou $q_{2}$ est impair.

On déduit de cette proposition le

Corollaire 2.2. L'homomorphisme caractéristique généralisé

$$
\Delta_{*}=\Delta_{*}(P): H\left(W(g, H)_{[(q+1) / 2]}\right) \longrightarrow H_{D R}(M)
$$

d'un G-fibré principal $F$-feuilletê $P \longrightarrow M$ admettant une connexion basique $\omega$ est indépendant de la connexion basique $\omega$ sur $P$ choisie, où $q$ est la codimension du feuilletage $F$ sur $M$.

De la même manière, on a la factorisation

$$
\Delta_{*}: H\left(W(g, H)_{[(q+1) / 2]}\right) \stackrel{p_{*}}{\longrightarrow} H\left(W(g, H)_{[q / 2]}\right) \longrightarrow H_{D R}(M),
$$

mais l'homomorphisme $\Delta_{*}=\Delta(\omega)_{*}: H\left(W(g, H)_{[q / 2]}\right) \longrightarrow H_{D R}(M)$ dépend, en général, du choix de la connexion basique $\omega$ sur $P$ si $q$ est impair.

\section{§. Calcul de la Cohomologie $H\left(W(g, H)_{I}\right)$}

Soient $\left(G_{i}, H_{i}\right), i=1,2$, des paires de groupes de Lie, $\left(g_{i}, h_{i}\right)$ leurs paires 
d'algèbres de Lie. On désigne par $(G, H)$ la paire de groupes de Lie $\left(G_{1} \times\right.$ $\left.G_{2}, H_{1} \times H_{2}\right)$ et par $(g, h)$ sa paire d'algèbres de Lie $\left(g_{1} \oplus g_{2}, h_{1} \oplus h_{2}\right)$. Etant donnée une paire $\left(q_{1}, q_{2}\right)$ d'entiers, avec $0 \leqq q_{1} \leqq q_{2}$, considérons l'algèbre de Weil relative tronquée $W(g, H)_{I}$. Pour simplifier le calcul de la cohomologie $H\left(W(g, H)_{I}\right)$, on suppose que les algèbres des polynômes symétriques invariants $I(G)$ et $I(g)$ soient canoniquement isomorphes, que $H$ ait un nombre fini de composantes connexes et que, pour chaque $i=1,2$, la paire d'algèbres de Lie $\left(g_{2}, h_{i}\right)$ soit une CS-paire (i.e. une paire réductive d'algèbres de Lie admettant une transgression $\tau_{2}$ pour $g_{2}$ telle que $\operatorname{Ker}\left(I\left(g_{i}\right)\right.$ $\left.\longrightarrow I\left(h_{\imath}\right)\right)=\operatorname{Idéal}\left(\tau_{i} \hat{P}_{i}\right) \subset I\left(g_{2}\right)$, où $\hat{P}_{\imath}$ désigne l'espace de Samelson de la paire $\left.\left(g_{i}, h_{2}\right)\right)$. Par conséquent, $(g, h)$ est aussi une CS-paire.

Avec les hypothèses précédentes, Carballés [2], en utilisant un certain isomorphisme (d'algèbres graduées)

$$
H\left(\hat{A}_{I}\right) \otimes_{I(G)} I(H) \stackrel{\cong}{\longrightarrow} H\left(W(g, H)_{I}\right),
$$

réduit le calcul de la cohomologie $H\left(W(g, H)_{l}\right)$ à celui de la cohomologie de la DG-algèbre tronquée

$$
\widehat{A}_{I}=\hat{A}\left(W(g)_{I}\right)=\wedge \hat{P} \otimes I(G)_{I} \cong \wedge \hat{P}_{1} \otimes \wedge \hat{P}_{2} \otimes\left(I\left(G_{1}\right) \otimes I\left(G_{2}\right)\right)_{I},
$$

où $\hat{P}$ (resp. $\widehat{P}_{i}$ ) est l'espace de Samelson de la paire $(g, h)$ (resp. de la paire $\left.\left(g_{i}, h_{2}\right)\right)$. Si, de plus, l'application de restriction $I(G) \longrightarrow I(H)$ est surjective, l'homomorphisme canonique $H\left(\widehat{A}_{I}\right) \stackrel{\cong}{\longrightarrow} H\left(\widehat{A}_{I}\right) \otimes_{I(G)} I(H)$ est un isomorphisme; par suite, on a donc

$$
H\left(\hat{A}_{I}\right) \cong H\left(\hat{A}_{I}\right) \otimes_{I(G)} I(H) \cong H\left(W(g, H)_{I}\right) .
$$

C'est par exemple le cas si $(G, H)=\left(G L\left(r_{1}\right) \times G L\left(r_{2}\right), O\left(r_{1}\right) \times O\left(r_{2}\right)\right)$. En particulier, pour $r_{1}=q_{1}$ et $r_{2}=q_{2}-q_{1}$, on vérifie que $\hat{A}_{I}$ est la DG-algèbre $W O_{I}$ utilisée par Cordero-Masa [3] dans la construction de l'homomorphisme caractéristique $\Delta_{*}=\lambda_{\left(F_{1}, F_{2}\right)}^{*}: H\left(W O_{I}\right) \longrightarrow H_{D R}(M)$ d'un sous-feuilletage $\left(F_{1}, F_{2}\right)$ de codimension $\left(q_{1}, q_{2}\right)$ sur $M$.

Nous calculons maintenant la cohomologie $H\left(\widehat{A}_{I}\right)$ comme suit. Soient $\left(g_{2}, h_{2}\right), i=1,2$, deux CS-paires d'algèbres de Lie de dimension finie sur un corps commutatif $\Re$ de caractéristique zéro ; on considère la CS-paire $(g, h)$ $=\left(g_{1} \oplus g_{2}, h_{1} \oplus h_{2}\right)$ d'algèbres de Lie. Soit $P_{g}=P_{g_{1}} \oplus P_{g_{2}}$ le sous-espace des éléments primitifs de $g$ (où $P_{g_{l}}$ désigne le sous-espace des éléments primitifs de $g_{2}$ ) et soit $\hat{P}=\hat{P}_{1} \oplus \hat{P}_{2} \subset P_{g}$ l'espace de Samelson de la paire $(g, h)$ (où $\hat{P}_{2}$ 
est l'espace de Samelson de la paire $\left.\left(g_{i}, h_{i}\right)\right)$. Alors on a $I\left(g_{1}\right) \cong \Re\left[c_{1}, c_{r_{1}}\right]$ avec $\operatorname{deg} c_{i} \leqq \operatorname{deg} c_{i+1}$ et $r_{1}=$ rang $g_{1}$, et de même, $I\left(g_{2}\right) \cong \Re\left[c_{1}^{\prime}, \cdots, c_{r_{2}}^{\prime}\right]$ avec deg $c_{i}{ }^{\prime} \leqq \operatorname{deg} c_{i+1}^{\prime}$ et $r_{2}=$ rang $g_{2}$. Par conséquent, une base de $P_{g_{1}}$ (resp. de $P_{g_{2}}$ ) est donnée par les éléments transgressifs avec $c_{1}, \cdots, c_{r_{1}}$ (resp. avec $c_{1}^{\prime}, \cdots$, $\left.c_{r_{2}}^{\prime}\right)$. Soient $r_{i}^{\prime}=\operatorname{dim} \hat{P}_{i}=\operatorname{rang} g_{i}-\operatorname{rang} h_{i}, i=1,2$, et $y_{1}, \cdots, y_{r_{1^{\prime}}}$ (resp. $y_{1}^{\prime}, \cdots$, $\mathrm{y}_{r_{2}}^{\prime}$ ) une base de $\hat{P}_{1}$ (resp. de $\widehat{P}_{2}$ ) telle que $y_{i}$ (resp. $\left.y_{i}^{\prime}\right)$ soit transgressif avec $c_{\alpha_{2}}$ pour $\alpha_{1}<\cdots<a_{r_{1}^{\prime}}$ (resp. avec $c_{\beta_{1}}^{\prime}$ pour $\beta_{1}<\cdots<\beta_{r_{2^{\prime}}}$. En particulier, si $h_{1}$ $=0\left(\right.$ resp. $\left.h_{2}=0\right)$ ), alors $\widehat{P}_{1}=P_{g_{1}}, r_{1}^{\prime}=r_{1}$ et $\alpha_{i}=i$ pour $i=1, \cdots, r_{1}$ (resp. $\widehat{P}_{2}=$ $P_{g_{2}}, r_{2}^{\prime}=r_{2}$ et $\beta_{i}=i$ pour $\left.i=1, \cdots, r_{2}\right)$. De la même manière, si $h_{1}=h_{2}=0$, alors $\hat{P}_{1}=P_{g_{1}}, \hat{P}_{2}=P_{g_{2}}, r_{1}^{\prime}=r_{1}, r_{2}^{\prime}=r_{2}, \alpha_{i}=i$ et $\beta_{i}=i$ pour tout $i$.

D'autre part, pour $0 \leqq q_{1} \leqq q_{2} \leqq \infty$, considérons l'idéal

$$
I=F^{2\left(q_{1}+1\right)} I(g)+F^{2\left(q_{2}+1\right)} I(g) \subset I(g) \cong I\left(g_{1}\right) \otimes I\left(g_{2}\right)
$$

(engendré par les sous-espaces $I^{2 i}\left(g_{1}\right) \otimes I^{2 j}\left(g_{2}\right)$ tels que $i>q_{1}$ ou $i+j>q_{2}$ ) et la DG-algèbre tronquée

$$
\begin{aligned}
\widehat{A}_{I}= & \widehat{A}\left(W(g)_{I}\right) \cong \bigwedge\left(y_{1}, \cdots, y_{r_{1}}\right) \otimes \wedge\left(y_{1}^{\prime}, \cdots, y_{r_{2}{ }^{\prime}}^{\prime}\right) \\
& \otimes\left(\Re\left[c_{1}, \cdots, c_{r_{1}}\right] \otimes \Re\left[c_{1}^{\prime}, \cdots, c_{r_{2}}^{\prime}\right]\right)_{I}
\end{aligned}
$$

dont la différentielle $d$ (de degré +1 ) est caractérisée par $d y_{i}=c_{a_{i}}, d c_{i}=0$, $d y_{i}{ }^{\prime}=c_{\beta_{i}}{ }^{\prime}$ et $d c_{i}{ }^{\prime}=0$ pour tout $i$.

Pour énoncer le résultat, nous avons besoin des notations suivantes:

$$
\begin{aligned}
& y_{(i)}=y_{i_{1}} \wedge \cdots \wedge y_{i_{s}} \text { pour } \quad(i)=\left(i_{1}, \cdots, i_{s}\right), \\
& 1 \leqq i_{1}<\cdots<i_{s} \leqq r_{1}^{\prime}, \text { si } s>0 \text {; } \\
& y_{(i)}=1 \text { pour }(i)=\emptyset \text { si } s=0 \text {; } \\
& y_{\left(i^{\prime}\right)}^{\prime}=y_{i_{1}}^{\prime} \wedge \cdots \wedge y_{i^{\prime} s^{\prime}}^{\prime} \text { pour }\left(i^{\prime}\right)=\left(i_{1}^{\prime}, \cdots, i_{s^{\prime}}^{\prime}\right) \text {, } \\
& 1 \leqq i_{1}^{\prime}<\cdots<i_{s^{\prime}}^{\prime} \leqq r_{2}^{\prime}, \text { si } s^{\prime}>0 \text {; } \\
& y_{\left(i^{\prime}\right)}^{\prime}=1 \text { pour }\left(i^{\prime}\right)=\emptyset \text { si } s^{\prime}=0 \text {; } \\
& c_{(j)}=c_{1}{ }^{j_{1}} \cdots c_{r_{1}}^{j r_{1}} \quad \text { pour }(j)=\left(j_{1}, \cdots, j_{r_{1}}\right), \quad 0 \leqq j_{i} ;
\end{aligned}
$$

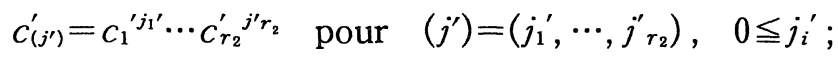

$$
\begin{aligned}
& z_{(i, j)}=y_{(i)} \otimes c_{(j)}, \quad z_{\left(i^{\prime}, j^{\prime}\right)}^{\prime}=y_{\left(i^{\prime}\right)}^{\prime} \otimes c_{\left(j^{\prime}\right)}^{\prime} \text {; } \\
& z_{\left(i, i^{\prime}, j, j^{\prime}\right)}=z_{(i, j)} \otimes z_{\left(i^{\prime}, j^{\prime}\right)}^{\prime}=y_{(i)} \otimes y_{\left(i^{\prime}\right)}^{\prime} \otimes c_{(j)} \otimes c_{\left(j^{\prime}\right)}^{\prime} ; \\
& 2 n_{i}=\operatorname{deg} c_{i}, \quad 2 p_{1}=\operatorname{deg} c_{(j)}=2 \sum_{i=1}^{r_{1}} j_{i} \cdot n_{i} ;
\end{aligned}
$$




$$
\begin{aligned}
& 2 n_{i}^{\prime}=\operatorname{deg} c_{i}^{\prime}, \quad 2 p_{2}=\operatorname{deg} c_{\left(\jmath^{\prime}\right)}^{\prime}=2 \sum_{i=1}^{r_{2}} j_{i}^{\prime} \circ n_{i}^{\prime}, \quad p=p_{1}+p_{2} ; \\
& i_{0}=i_{1} \quad \text { et } \quad n_{20}=n_{\alpha_{11}} \quad \text { si } s>0, \quad i_{0}=n_{i_{0}}=\infty \text { si } s=0 \text {; } \\
& i_{0}^{\prime}=i_{1}^{\prime} \quad \text { et } \quad n_{i_{0}}^{\prime}=n_{\beta_{2_{1}}}^{\prime} \text { si } s^{\prime}>0, \quad i_{0}^{\prime}=n_{{ }^{\prime} 0^{\prime}}^{\prime}=\infty \quad \text { si } \quad s^{\prime}=0 \text {; } \\
& j_{0}=\min A, \quad \text { et } n_{j_{0}}=n_{\alpha_{0}} \text { si } A_{\jmath} \neq \emptyset, \quad j_{0}=n_{j_{0}}=\infty \text { si } A_{j}=\emptyset \text {; } \\
& j_{0}^{\prime}=\min A_{j^{\prime}}^{\prime} \quad \text { et } \quad n_{j^{\prime}}^{\prime}=n_{\beta, 6}^{\prime} \text { si } \quad A_{j^{\prime}}^{\prime} \neq \emptyset \text {, } \\
& j_{0}^{\prime}=n_{j 6}^{\prime}=\infty \text { si } A_{j^{\prime}}^{\prime}=\emptyset \text {, }
\end{aligned}
$$

où $A_{\mathrm{u}}$ (resp. $A_{j^{\prime}}^{\prime}$ ) désigne l'ensemble des éléments $i=1, \cdots, r_{1}^{\prime}$ tels que $j_{\alpha_{i}}>$ 0 (resp. des éléments $i=1, \cdots, r_{2}^{\prime}$ tels que $j_{\beta_{2}}>0$ ).

Nous obtenons alors le résultat suivant.

Théorème 3.1. Une $\Re$-base de $H\left(\hat{A}_{I}\right)$ est fournie par les classes de cohomologie des cocycles monômiaux $z_{\left(2,2^{\prime},, j^{\prime}\right)}$ vérifiant les conditions suivantes :

(1) $0 \leqq p_{1} \leqq q_{1}, 0 \leqq p \leqq q_{2}, 0 \leqq s \leqq r_{1}^{\prime}$ et $0 \leqq s^{\prime} \leqq r_{2}^{\prime}$;

(2) $n_{i_{0}}+p_{1} \geqq q_{1}+1$ ou $n_{i_{0}}+p \geqq q_{2}+1$, et $n_{i_{0^{\prime}}}+p \geqq q_{2}+1$ (condition de cocycle);

(3) cette condition est énoncée comme suit:

(a) $n^{\prime}{ }^{\prime} 0^{\prime}<n_{j_{0}}$ et $i_{0}{ }^{\prime} \leqq j_{0}^{\prime}$ si $n_{i_{0}}>n^{\prime}{ }^{\prime} 0^{\prime}$;

(b) $i_{0} \leqq j_{0}$ et $n_{i_{0}} \leqq n_{j_{0^{\prime}}}^{\prime}$ si $n_{i_{0}} \leqq n_{i_{0^{\prime}}^{\prime}}^{\prime}$ et $n_{{ }_{0}}+p_{1}<q_{1}+1$;

(c) $i_{0} \leqq j_{0}$ et $i_{0}^{\prime} \leqq j_{0}^{\prime}$ si $n_{i_{0}} \leqq n^{\prime} i_{0^{\prime}}$ et $n_{i_{0}}+p_{1} \geqq q_{1}+1$.

Démonstration. Il est clair que l'ensemble $C$ des éléments $z_{\left(i, i^{\prime}, j, j^{\prime}\right)}$ tels que $0 \leqq p_{1} \leqq q_{1}, 0 \leqq p \leqq q_{2}, 0 \leqq s \leqq r_{1}^{\prime}$ et $0 \leqq s^{\prime} \leqq r_{2}^{\prime}$; constitue une $\Re$-base de $\widehat{A}_{I}$. Soient $C_{a}, C_{b}$ et $C_{c}$ les ensembles des $z_{\left(i, i^{\prime}, j, j^{\prime}\right)} \in C$ satisfaisant respectivement aux conditions suivantes:

(a) $n_{i_{0}}>n_{i_{0}{ }^{\prime}}^{\prime}, n_{i_{0^{\prime}}}^{\prime}<n_{j_{0}}$ et $i_{0}^{\prime} \leqq j_{0}^{\prime}$;

(b) $n_{i_{0}} \leqq n_{i^{\prime}}^{\prime}, n_{i_{0}}+p_{1}<q_{1}+1, i_{0} \leqq j_{0}$ et $n_{i_{0}} \leqq n^{\prime}{ }_{\jmath^{\prime}}$;

(c) $n_{i_{0}} \leqq n^{\prime}{ }_{i_{0}}{ }^{\prime}, n_{i_{0}}+p_{1} \geqq q_{1}+1, i_{0} \leqq j_{0}$ et $i_{0}{ }^{\prime} \leqq j_{0}^{\prime}$

(en particulier, on a donc $C_{a} \cap C_{b}=C_{a} \cap C_{c}=C_{b} \cap C_{c}=\emptyset$ ). Posons $C_{a}{ }^{\prime}=C_{a}$ $-C_{a}{ }^{\prime \prime}, C_{b}{ }^{\prime}=C_{b}-C_{b}{ }^{\prime \prime}$ et $C_{c}{ }^{\prime}=C_{c}-C_{c}{ }^{\prime \prime}$, où $C_{a}{ }^{\prime \prime}, C_{b}{ }^{\prime \prime}$ et $C_{c}{ }^{\prime \prime}$ désignent les ensembles des $z_{\left(2,2^{\prime}, j, j^{\prime}\right)}$ appartenant respectivement à $C_{a}, C_{b}$ et $C_{c}$ tels que soient des cocycles (c'est-à-dire satisfaisant respectivement aux conditions suivantes: $\left.\quad n_{i_{0^{\prime}}}^{\prime}+p \geqq q_{2}+1 ; \quad n_{i_{0}}+p \geqq q_{2}+1 ; \quad n_{i_{0^{\prime}}}^{\prime}+p \geqq q_{2}+1\right)$. Considérons maintenant les sous-ensembles de $C$ donnés par $C_{1}{ }^{\prime}=C_{a}{ }^{\prime} \cup C_{b}{ }^{\prime} \cup C_{c}{ }^{\prime}, C_{1}{ }^{\prime \prime}=$ $C_{a}{ }^{\prime \prime} \cup C_{b}{ }^{\prime \prime} \cup C_{c}{ }^{\prime \prime}, C_{1}=C_{1}{ }^{\prime} \cup C_{1}{ }^{\prime \prime}=C_{a} \cup C_{b} \cup C_{c}, C_{2}=C-C_{1}$ et $C_{2}{ }^{\prime}=C_{2}-C_{2}{ }^{\prime \prime}$, 
où $C_{2}{ }^{\prime \prime}$ est l'ensemble des $z_{\left(i, i^{\prime}, j, j^{\prime}\right)} \in C_{2}$ tels que $n_{i_{0}} \leqq n_{i^{\prime}}^{\prime}, n_{i_{0}}+p_{1} \geqq q_{1}+1, i_{0} \leqq$ $j_{0}, \quad n_{i_{0}} \leqq n_{j_{0^{\prime}}}^{\prime}$ et $j_{0}^{\prime}<i_{0}^{\prime}$. Par conséquent, $C_{1}^{\prime \prime}$ est l'ensemble des $z_{\left(i, i^{\prime}, j, j^{\prime}\right)}$ satisfaisant aux conditions (1), (2) et (3).

D'autre part, étant donné un élément $z_{\left(i, i^{\prime}, j, j^{\prime}\right)} \in C$, on a alors

$$
d z_{\left(i, i^{\prime}, j, j^{\prime}\right)}=\sum_{t=1}^{s}(-1)^{t+1} z_{t}+\sum_{t^{\prime}=1}^{s^{\prime}}(-1)^{s+t^{\prime}+1} z_{t^{\prime}}^{\prime}
$$

où $z_{t}$ et $z_{t^{\prime}}^{\prime}$ désignent respectivement les éléments

$$
\begin{aligned}
& y_{i_{1}} \wedge \cdots \wedge \bar{y}_{i_{t}} \wedge \cdots \wedge y_{i_{s}} \otimes y_{\left(i^{\prime}\right)}^{\prime} \otimes c_{\alpha_{i t}} \cdot c_{(j)} \otimes c^{\prime}{ }_{\left(j^{\prime}\right)} \text { et }
\end{aligned}
$$

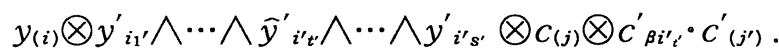

Supposons maintenant que $z=z_{\left(i, i^{\prime}, j, j^{\prime}\right)} \in C_{1}{ }^{\prime}=C_{a}{ }^{\prime} \cup C_{b}{ }^{\prime} \cup C_{c}{ }^{\prime}$; alors on voit que $d z \neq 0 \in \widehat{A}_{I}$ et que les éléments $z_{t}, 1 \leqq t \leqq s$, et $z_{t^{\prime}}, 1 \leqq t^{\prime} \leqq s^{\prime}$, vérifient les conditions suivantes :

(a') $z_{1}^{\prime} \in C_{2}^{\prime}$ et les $z_{t}, 1 \leqq t \leqq s$, et $z_{t^{\prime}}^{\prime}, 2 \leqq t^{\prime} \leqq s^{\prime}$, différents de $0 \in \widehat{A_{1}}$ appartiennent à $C_{a}$ si $z \in C_{a}{ }^{\prime}$;

( $\left.\mathrm{b}^{\prime}\right) \quad z_{1} \in C_{2}^{\prime}$ et les $z_{t}, 2 \leqq t \leqq s$, et $z_{t^{\prime}}^{\prime}, 1 \leqq t^{\prime} \leqq s^{\prime}$, différents de $0 \in \widehat{A}_{I}$ appartiennent respectivement à $C_{b} \cup C_{c} \cup C_{2}{ }^{\prime \prime}$ et $C_{b}$ si $z \in C_{b}{ }^{\prime}$;

(c') $z_{1}^{\prime} \in C_{2}^{\prime \prime}, z_{t}=0 \in \widehat{A}_{I}, 1 \leqq t \leqq s$, et les $z_{t^{\prime}}^{\prime}, 2 \leqq t^{\prime} \leqq s^{\prime}$, différents de $0 \in \widehat{A}_{I}$ appartiennent à $C_{c}$ si $z \in C_{c}{ }^{\prime}$.

Il s'ensuit que l'application

$$
\delta: C_{1}{ }^{\prime}=C_{a}{ }^{\prime} \cup C_{b}{ }^{\prime} \cup C_{c}{ }^{\prime} \longrightarrow C_{2}=C_{2}{ }^{\prime} \cup C_{2}{ }^{\prime \prime}
$$

définie par $\delta z=z_{1}{ }^{\prime}$ pour $z \in C_{a}{ }^{\prime} \cup C_{c}{ }^{\prime}$ et $\delta z=z_{1}$ pour $z \in C_{b}{ }^{\prime}$, est bijective. Compte tenu des conditions $\left(a^{\prime}\right),\left(b^{\prime}\right)$ et $\left(c^{\prime}\right)$, il en résulte que l'application $d_{1 c^{\prime}}: C_{1}^{\prime} \longrightarrow d\left(C_{1}^{\prime}\right)$ est bijective et que les ensembles $d\left(C_{1}^{\prime}\right), C_{1} \cup d\left(C_{1}^{\prime}\right)$ (avec $C_{1} \cap d\left(C_{1}{ }^{\prime}\right)=\emptyset$ ) et $C_{1}{ }^{\prime \prime} \cup d\left(C_{1}{ }^{\prime}\right)$ sont respectivement $\Omega$-bases de $d \bar{A}_{I}, \bar{A}_{I}$ et $\operatorname{Ker} d$. Par suite, on a alors

$$
\widehat{A}_{I}=\widetilde{C}_{1} \oplus d \widehat{A}_{I}=\widetilde{C}_{1}{ }^{\prime} \oplus \widetilde{C}_{1}{ }^{\prime \prime} \oplus d \widehat{A}_{I} \quad \text { et } \quad \operatorname{Ker} d=\widetilde{C}_{1}{ }^{\prime \prime} \oplus d \widehat{A}_{I}
$$

où $\widetilde{C}_{1}, \widetilde{C}_{1}^{\prime}$ et $\widetilde{C}_{1}^{\prime \prime}$ désignent les $\Re$-sous-espaces de $\hat{A}_{I}$ engendrés respectivement par les ensembles $C_{1}, C_{1}^{\prime}$ et $C_{1}^{\prime \prime}$. Ceci prouve que la projection canonique $\operatorname{Ker} d \longrightarrow H\left(\hat{A}_{I}\right)=\operatorname{Ker} d / d \widehat{A}_{I}$ induit un isomorphisme $\Re$-linéaire $\widetilde{C}_{1}^{\prime \prime} \cong H\left(\widehat{A}_{I}\right)$, d'où le théorème.

Remarques. 1) Le résultat précédent généralise celui de KamberTondeur [8], Théorème 5.110 (cf. aussi Masa [10]). Par conséquent, le Théorème 3.1 est une généralisation du théorème de Vey [7], p.383. 
2) Les classes des $z_{(i, \phi, j, 0)}=z_{(i, j)} \otimes 1 \in C_{1}^{\prime \prime}$ avec $s^{\prime}=p_{2}=0$ (resp. des $z_{\left(\phi, i^{\prime}, 0, j^{\prime}\right)}=1 \otimes z_{\left(i^{\prime}, j^{\prime}\right)}^{\prime} \in C_{1}^{\prime \prime}$ avec $\left.s=p_{1}=0\right)$ constituent une base de l'image de l'homomorphisme injectif canonique $H\left(\hat{A}\left(W\left(g_{1}\right)_{q_{1}}\right)\right) \longrightarrow H\left(\widehat{A}_{I}\right)$ (resp. $\left.H\left(\hat{A}\left(W\left(g_{2}\right)_{q_{2}}\right)\right) \longrightarrow H\left(\hat{A}_{I}\right)\right)$. De même, une base de l'image de l'homomorphisme canonique $H\left(\hat{A}\left(W(g)_{q_{2}}\right)\right) \longrightarrow H\left(\widehat{A}_{I}\right)$ est fournie par les classes des $z_{\left(2,2^{\prime}, j, j^{\prime}\right)} \in C_{1}$ " tels que $n_{20}+p \geqq q_{2}+1$. En particulier, si $q_{1}=q_{2}=$ $q \geqq 0$, alors $\hat{A}\left(W(g)_{q}\right)=\hat{A}_{I}$ et les éléments de la base de Vey de $H\left(\widehat{A}\left(W(g)_{q}\right)\right)$ coïncident (à un signe près) avec les éléments de la base de $H\left(\widehat{A}_{I}\right)$ donnée dans le Théorème 3.1. D'autre part, pour $q_{1}=0$ ou $q_{2}=\infty$, on a donc $H\left(\hat{A}_{I}\right) \cong H\left(\widehat{A}\left(W\left(g_{1}\right)_{q_{1}}\right)\right) \otimes H\left(\widehat{A}\left(W\left(g_{2}\right)_{q_{2}}\right)\right)$.

3) Les classes des $z_{\left(\phi, \phi,, J^{\prime}\right)}=1 \otimes 1 \otimes c_{(\jmath)} \otimes c^{\prime}\left(\jmath^{\prime}\right) \in C_{1}^{\prime \prime}, \quad s=s^{\prime}=0$, constituent une $\Re$-base de l'algèbre des classes caractéristiques universelles primaires (induites par l'homomorphisme canonique $I(g) \longrightarrow H\left(\hat{A}_{I}\right)$ ). De même, les classes des $z_{\left(2,2^{\prime},,,^{\prime}\right)} \in C_{1}{ }^{\prime \prime}$ tels que $s>0$ ou $s^{\prime}>0$ constituent une $\Re$-base de l'algèbre des classes caractéristiques universelles secondaires ; de plus, les conditions suivantes sont vérifiées:

(a) $2 q_{1}+1 \leqq \operatorname{deg} z_{\left(2, i^{\prime}, 0, \nu^{\prime}\right)} \leqq 2 q_{2}+m_{1}$ pour $s>0$ et $s^{\prime}=0$ (si, de plus, $p_{2}=0$, on a deg $\left.z_{\left(2,2^{\prime},,,^{\prime}\right)} \leqq 2 q_{1}+m_{1}\right)$, où $m_{1}=\operatorname{dim} g_{1}$;

(b) $2 q_{2}+1 \leqq \operatorname{deg} z_{\left(2,2^{\prime},,, j^{\prime}\right)} \leqq 2 q_{2}+m_{2}$ pour $s=0$ et $s^{\prime}>0$, où $m_{2}=\operatorname{dim} g_{2}$;

(c) $2 q_{2}+2 \leqq \operatorname{deg} z_{\left(2, i^{\prime}, 0, v^{\prime}\right)} \leqq 2 q_{2}+m$ pour $s>0$ et $s^{\prime}>0$, où $m=m_{1}+m_{2}=$ $\operatorname{dim} g$.

4) Pour $0<q_{1}<q_{2}<\infty$, les classes secondaires des $z_{\left(2,2^{\prime}, 0, j^{\prime}\right)} \in C_{c}{ }^{\prime \prime}$ (avec $s>0$ et $\left.s^{\prime}>0\right)$ tels que $n_{i_{0}}+p_{1} \geqq q_{1}+1, n_{i_{0}}+p<q_{2}+1, n_{{ }^{\prime} 0^{\prime}}+p \geqq q_{2}+1$ et $n^{\prime}{ }^{\prime}{ }^{\prime}+p_{2}<q_{2}+1$ (en particulier, $\left.n_{20}<n^{\prime}{ }^{\prime} 0^{\prime}\right)$, ne sont pas induites dans $H\left(\widehat{A}_{I}\right)$ par celles de $H\left(\hat{A}\left(W\left(g_{1}\right)_{q_{1}}\right)\right) \otimes H\left(\hat{A}\left(W\left(g_{2}\right)_{q_{2}}\right)\right)$ ou $H\left(\hat{A}\left(W(g)_{q_{2}}\right)\right)$.

Définition 3.2. La $\Re$-base de $H\left(\widehat{A}_{I}\right)$ fournie par les classes des $z_{\left(i, 1^{\prime}, j, j^{\prime}\right)}$ $\in C_{1}{ }^{\prime \prime}$ satisfaisant aux conditions (1), (2) et (3) du Théorème 3.1 sera appelée la base de Vey de $H\left(\hat{A}_{l}\right)$.

Exemple 1. Pour la paire $(g, h)=\left(g l\left(r_{1}\right) \oplus g l\left(r_{2}\right), \quad s o\left(r_{1}\right) \oplus s o\left(r_{2}\right)\right)$ d'algèbres de Lie de la paire $(G, H)=\left(G L\left(r_{1}\right) \times G L\left(r_{2}\right), O\left(r_{1}\right) \times O\left(r_{2}\right)\right)$ de groupes de Lie et $0 \leqq q_{1} \leqq q_{2} \leqq \infty$, considérons la DG-algèbre tronquée $\widehat{A}_{I} \cong$ $\wedge\left(y_{1}, y_{3}, \cdots, y_{r_{1}}\right) \otimes \wedge\left(y_{1}^{\prime}, y_{3}^{\prime}, \cdots, y_{r_{2}}^{\prime}\right) \otimes\left(\Re\left[c_{1}, c_{2}, \cdots, c_{r_{1}}\right] \otimes \Re\left[c_{1}^{\prime}, c_{2}^{\prime}, \cdots, c^{\prime} r_{2}\right]\right)_{1}$ dont la différentielle $d$ (de degré +1 ) est donnée par

$$
d y_{\imath}=c_{\imath}, \quad d c_{2}=0, \quad d y_{\imath}^{\prime}=c_{\imath}^{\prime} \text { et } \quad d c_{\imath}^{\prime}=0 \text { pour tout } i,
$$

où les éléments $y_{2}$ (resp. $\left.y_{2}^{\prime}\right)$ de degrés $2 i-1$ sont les suspensions des 
polynômes de Chern $c_{i} \in I\left(G L\left(r_{1}\right)\right) \cong \Re\left[c_{1}, c_{2}, \cdots, c_{r_{1}}\right]\left(\right.$ resp. $c_{i}^{\prime} \in I\left(G L\left(r_{2}\right)\right) \cong$ $\left.\Re\left[c_{1}^{\prime}, c_{2}^{\prime}, \cdots, c_{r_{2}}^{\prime}\right]\right)$ de degrés $2 i$, avec $i$ impair, et où $r_{i}^{\prime}=2\left[\left(r_{i}+1\right) / 2\right]-1, i=$ 1, 2. Soient maintenant

$$
\begin{aligned}
& z_{\left(i, i^{\prime}, j, j^{\prime}\right)}=y_{(i)} \otimes y_{{ }_{\left(i^{\prime}\right)}^{\prime}}^{\prime} \otimes c_{(j)} \otimes c^{\prime}{ }_{\left(j^{\prime}\right)}=y_{i_{1}} \wedge \cdots \wedge y_{i_{s}} \otimes y_{i^{\prime}}^{\prime} \wedge \cdots \wedge y_{i^{\prime} s}^{\prime} \\
& \otimes c_{1}{ }^{j_{1}} \cdots c_{r_{1}}^{j_{1}} \otimes c_{1}^{\prime{ }^{\prime}{ }_{1}} \cdots c_{r_{2}}^{\prime j^{\prime} r_{2}}
\end{aligned}
$$

les cochaînes de $\hat{A}_{I}$ telles que

$$
\begin{aligned}
& 1 \leqq i_{1}<\cdots<i_{s} \leqq r_{1}^{\prime} \text { avec } i_{k} \text { impair pour } s>0, \\
& y_{(i)}=1 \text { pour } s=0 ; \\
& 1 \leqq i_{1}^{\prime}<\cdots<i_{s^{\prime}}^{\prime} \leqq r_{2}^{\prime} \text { avec } i_{k}^{\prime} \text { impair pour } s^{\prime}>0, \\
& y_{\left(i^{\prime}\right)}^{\prime}=1 \text { pour } s^{\prime}=0 ; \\
& 2 p_{1}=\operatorname{deg} c_{(j)}=2 \sum_{i=1}^{r_{1}} j_{i} \cdot i, \quad j_{i} \geqq 0, \quad 2 p_{2}=\operatorname{deg} c^{\prime}\left(j^{\prime}\right)=2 \sum_{i=1}^{r_{2}} j_{i}^{\prime} \cdot i, \\
& j_{i}^{\prime} \geqq 0, \quad p=p_{1}+p_{2} ; \\
& i_{0}=i_{1} \text { pour } s>0, \quad i_{0}=\infty \text { pour } s=0, \\
& i_{0}^{\prime}=i_{1}^{\prime} \text { pour } s^{\prime}>0, \quad i_{0}^{\prime}=\infty \text { pour } s^{\prime}=0 ; \\
& j_{0}=\min A_{j} \text { pour } A_{j} \neq \emptyset, \quad j_{0}=\infty \text { pour } A_{j}=\emptyset, \\
& j_{0}^{\prime}=\min A_{j^{\prime}}^{\prime} \text { pour } A_{j^{\prime}}^{\prime} \neq \emptyset, \quad j_{0}^{\prime}=\infty \text { pour } A_{j^{\prime}}^{\prime}=\emptyset,
\end{aligned}
$$

où $A_{j}$ (resp. $A_{j^{\prime}}^{\prime}$ ) désigne l'ensemble des $i=1,3, \cdots, r_{1}^{\prime}$ tels que $j_{i}>0$ (resp. des $i=1,3, \cdots, r_{2}^{\prime}$ tels que $\left.j_{i}^{\prime}>0\right)$. Alors, d'après le Théorème 3.1, la base de Vey de $H\left(\widehat{A}_{I}\right) \cong H\left(W(g, H)_{I}\right)$ est constituée par les classes de cohomologie des cocycles monômiaux $z_{\left(i, i^{\prime}, j, j^{\prime}\right)}$ satisfaisant aux conditions suivantes :

(1) $0 \leqq p_{1} \leqq q_{1}, \quad 0 \leqq p \leqq q_{2}, \quad 0 \leqq s \leqq\left[\left(r_{1}+1\right) / 2\right]$ et $0 \leqq s^{\prime} \leqq\left[\left(r_{2}+1\right) / 2\right]$;

(2) $i_{0}+p_{1} \geqq q_{1}+1$ ou $i_{0}+p \geqq q_{2}+1$, et $i_{0}^{\prime}+p \geqq q_{2}+1$ (condition de cocycle) ;

(3) cette condition est énoncée comme suit:

(a) $i_{0}^{\prime}<j_{0}$ et $i_{0}^{\prime} \leqq j_{0}^{\prime}$ si $i_{0}>i_{0}^{\prime}$;

(b) $i_{0} \leqq j_{0}$ et $i_{0} \leqq j_{0}^{\prime}$ si $i_{0} \leqq i_{0}^{\prime}$ et $i_{0}+p_{1}<q_{1}+1$;

(c) $i_{0} \leqq j_{0}$ et $i_{0}^{\prime} \leqq j_{0}^{\prime}$ si $i_{0} \leqq i_{0}^{\prime}$ et $i_{0}+p_{1} \geqq q_{1}+1$.

En particulier, les classes des cocycles suivants seront des classes caractéristiques secondaires de degré minimum (pour $q_{2}<\infty$ ):

(i) $y_{1} \otimes c_{1}{ }^{q_{1}}=y_{1} \otimes 1 \otimes c_{1}{ }^{q_{1}} \otimes 1$ de degré $2 q_{1}+1$;

(ii) $y_{1}^{\prime} \otimes c_{1}^{\prime} q_{2}=1 \otimes y_{1}^{\prime} \otimes 1 \otimes c_{1}{ }^{\prime} q_{2}$ et $y_{1} \otimes c_{1}{ }^{j} c_{1}{ }^{\prime} q_{2-j}=y_{1} \otimes 1 \otimes c_{1}{ }^{j} \otimes c_{1}{ }^{\prime} q_{2-j}$ (ou son 


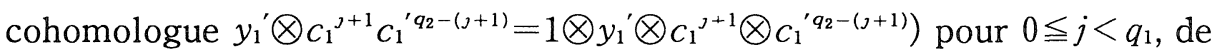
degré $2 q_{2}+1$;

(iii) $y_{1} \wedge y_{1}^{\prime} \otimes c_{1}{ }^{j} c_{1}{ }^{\prime} q_{2}-j=y_{1} \otimes y_{1}^{\prime} \otimes c_{1}{ }^{j} \otimes c_{1}{ }^{q_{2}-j}$ pour $0 \leqq j \leqq q_{1}$, de degré $2 q_{2}+2$.

Ainsi, pour $r_{1}=q_{1}$ et $r_{2}=q_{2}-q_{1} \geqq 0$ (avec $\left.q_{2}<\infty\right)$, on a obtenu la base de Vey de l'algèbre $H\left(\widehat{A}_{I}\right)=H\left(W O_{I}\right)$ des classes caractéristiques universelles pour les sous-feuilletages de codimension $\left(q_{1}, q_{2}\right)$.

De même, pour $r_{1}=r_{2}=1$ et $q_{2}<\infty$, les classes caractéristiques secondaires de degré minimum des cocycles (i), (ii) et (iii) constituent la base de Vey de

$$
\begin{aligned}
& H^{+}\left(W(g l(1) \oplus g l(1), \quad O(1) \times O(1))_{I}\right) \\
& \quad=H^{+}\left(W(g l(1) \oplus g l(1))_{I}\right)=H^{+}\left(\wedge\left(y_{1}\right) \otimes \wedge\left(y_{1}{ }^{\prime}\right) \otimes\left(\Re\left[c_{1}\right] \otimes \Re\left[c_{1}^{\prime}\right]\right)_{I}\right) .
\end{aligned}
$$

Deefinition 3.3. Les classes secondaires de degré minimum $\left[y_{1} \otimes c_{1}{ }^{q_{1}}\right]$, $\left[y_{1}^{\prime} \otimes c_{1}^{j} c_{1}{ }^{q_{2}-\jmath}\right], \quad\left[y_{1} \wedge y_{1}{ }^{\prime} \otimes c_{1}{ }^{j} c_{1}{ }^{q_{2}-j}\right] \in H^{+}\left(W(g l(1) \oplus g l(1))_{I}\right)$ pour $0 \leqq j \leqq q_{1}$, seront appelées les classes de Godbillon-Vey (universelles) pour les sousfeuilletages de codimension $\left(q_{1}, q_{2}\right)$.

Plus précisément, on verra au $\S 5$ que de telles classes nous permettent d'obtenir des classes caractéristiques secondaires pour les sous-feuilletages de codimension $\left(q_{1}, q_{2}\right)$, en utilisant des techniques similaires à celles utilisées par Godbillon-Vey [5] pour un feuilletage. Par exemple, si $\left(F_{1}\right.$, $F_{2}$ ) est un sous-feuilletage de codimension $\left(q_{1}, q_{2}\right)$ sur $M$, la classe de Godbillon-Vey de $F_{1}$ (resp. de $F_{2}$ ) est donnée par $\Delta_{*}\left[y_{1} \otimes C_{1}{ }^{q_{1}}\right] \in H_{D R}^{2 q_{1}+1}(M)$ (resp. par

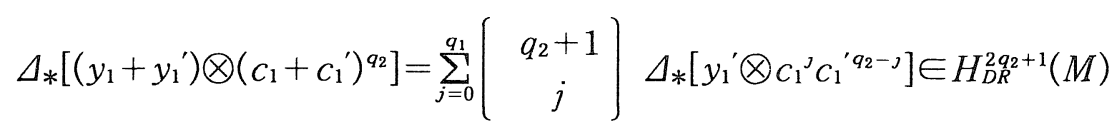

pour $\left.q_{1}<q_{2}\right)$; d'autre part, les classes de Godbillon-Vey

$$
\Delta_{*}\left[y_{1} \wedge y_{1}^{\prime} \otimes c_{1}{ }^{j} c_{1}{ }^{q_{2}-j}\right] \in H_{D R}^{2 q_{2}+2}(M), \quad 0 \leqq j \leqq q_{1},
$$

non nulles sont, en général (pour $0<q_{1}<q_{2}<\infty$ ), des nouveaux invariants caractéristiques secondaires du sous-feuilletage $\left(F_{1}, F_{2}\right)$ qui ne peuvent pas s'obtenir en considérant séparément les feuilletages de la paire.

Exemple 2. Prenons $(G, H)=\left(G L\left(r_{1}\right) \times G L\left(r_{2}\right), \quad S O\left(r_{1}\right) \times S O\left(r_{2}\right)\right)$. Compte tenu de l'isomorphisme $H\left(W(g, H)_{I}\right) \cong H\left(\widehat{A}_{I}\right) \otimes_{I(G)} I(H)$, avec $0 \leqq q_{1}$ $\leqq q_{2} \leqq \infty$, on a donc 


$$
H\left(W(g, H)_{I}\right) \cong\left\{\begin{array}{l}
H\left(\hat{A}_{I}\right) \quad \text { pour } r_{1}=2 m-1 \text { et } r_{2}=2 n-1 \\
\left(H\left(\hat{A}_{I}\right) \otimes \Re\left[e_{m}\right]\right) /\left(c_{2 m}-e_{m}{ }^{2}\right) \quad \text { pour } r_{1}=2 m \text { et } r_{2}=2 n-1 \\
\left(H\left(\hat{A}_{I}\right) \otimes \Re\left[e_{n}^{\prime}\right]\right) /\left(c^{\prime}{ }_{2 n}-e_{n}{ }^{\prime 2}\right) \quad \text { pour } r_{1}=2 m-1 \text { et } r_{2}=2 n \\
\left(H\left(\hat{A}_{I}\right) \otimes \Re\left[e_{m}\right] \otimes \Re\left[e_{n}^{\prime}\right]\right) /\left(c_{2 m}-e_{m}{ }^{2}, c_{2 n}{ }^{\prime}-e_{n}{ }^{\prime 2}\right) \\
\text { pour } r_{1}=2 m \text { et } r_{2}=2 n,
\end{array}\right.
$$

où la cohomologie $H\left(\widehat{A}_{I}\right)$ a été calculée dans l'Exemple 1 , et où $e_{m} \in$ $I^{2 m}\left(S O\left(r_{1}\right)\right)$ (resp. $\left.e_{n}^{\prime} \in I^{2 n}\left(S O\left(r_{2}\right)\right)\right)$ est le polynôme de Pfaff pour $r_{1}=2 m$ (resp. pour $r_{2}=2 n$ ).

Remarque. Le calcul de la cohomologie $H\left(W\left(u\left(r_{1}\right) \oplus u\left(r_{2}\right), O\left(r_{1}\right) \times\right.\right.$ $\left.\left.O\left(r_{2}\right)\right)_{I}\right)$ (resp. $\left.H\left(W\left(u\left(r_{1}\right) \oplus u\left(r_{2}\right), S O\left(r_{1}\right) \times S O\left(r_{2}\right)\right)_{I}\right)\right)$ est identique à celui de $H\left(W\left(g l\left(r_{1}\right) \oplus g l\left(r_{2}\right), O\left(r_{1}\right) \times O\left(r_{2}\right)\right)_{I}\right)$ (resp. de $H\left(W\left(g l\left(r_{1}\right) \oplus g l\left(r_{2}\right)\right.\right.$, $\left.\left.\left.S O\left(r_{1}\right) \times S O\left(r_{2}\right)\right)_{I}\right)\right)$.

De la même manière, nous calculons les cohomologies $H\left(W\left(g l\left(r_{1}\right) \oplus g l\left(r_{2}\right)\right)_{I}\right), H\left(W\left(u\left(r_{1}\right) \oplus u\left(r_{2}\right)\right)_{I}\right)$ et $H\left(W\left(g l\left(r_{1}, \mathfrak{S}\right) \oplus g l\left(r_{2}\right.\right.\right.$, $\left.(5))_{I}\right)$. Ainsi, nous obtenons en particulier la base de Vey de l'algèbre $H^{+}\left(W_{I}\right) \cong H^{+}\left(W\left(g l\left(q_{1}\right) \oplus g l\left(q_{2}-q_{1}\right)\right)_{I}\right)$ des classes caractéristiques universelles secondaires pour les sous-feuilletages de codimension $\left(q_{1}, q_{2}\right)$ à fibré normal trivialisé.

Soit maintenant $\hat{A}_{I}$ la DG-algèbre considérée dans le Théorème 3.1. Alors, par un raisonnement identique à celui qui a été utilisé dans la démonstration de ce théorème, on obtient le résultat suivant.

Théorème 3.4. Une $\Re$-base de $H\left(\widehat{A}_{I}\right)$ est fournie par les classes de cohomologie des cocycles monômiaux $z_{\left(i, i^{\prime}, j, j^{\prime}\right)}$ vérifiant les conditions $(1)$ et (2) du Théorème 3.1, et la condition suivante:

$\left(3^{\prime}\right)$ cette condition est énonciée comme suit:

(a') $\quad n_{i_{0^{\prime}}^{\prime}} \leqq n_{j_{0}}$ et $i_{0}^{\prime} \leqq j_{0}^{\prime}$ si $n_{i_{0}} \geqq n_{i_{0^{\prime}}^{\prime}}^{\prime}$;

(b') $i_{0} \leqq j_{0}$ et $n_{i_{0}}<n_{j_{0^{\prime}}}^{\prime}$ si $n_{i_{0}}<n_{i_{0^{\prime}}}^{\prime}$ et $n_{i_{0}}+p_{1}<q_{1}+1$;

(c') $i_{0} \leqq j_{0}$ et $i_{0}^{\prime} \leqq j_{0}^{\prime}$ si $n_{i_{0}}<n_{i_{0^{\prime}}^{\prime}}^{\prime}$ et $n_{i_{0}}+p_{1} \geqq q_{1}+1$.

\section{§ 4. Quelques Propriétés des Sous-feuilletages. Classes Caractéristiques}

Dans ce paragraphe nous étudions quelques propriétés des sousfeuilletages. En particulier, nous donnons certaines interprétations géométriques des classes de Godbillon-Vey d'un sous-feuilletage.

Soit $\left(F_{1}, F_{2}\right)$ un sous-feuilletage de codimension $\left(q_{1}, q_{2}\right)$ sur une variété 
différentiable $M$ de dimension $n$, et soit $d=q_{2}-q_{1} \geqq 0$. Considérons le fibré normal $Q_{1} \oplus Q_{0}=\left(T M / F_{1}\right) \oplus\left(F_{1} / F_{2}\right)$ (resp. $\left.Q_{2}=T M / F_{2}\right)$ de $\left(F_{1}, F_{2}\right)$ (resp. de $\left.F_{2}\right)$. D'après Cordero-Masa [3], les fibrés vectoriels isomorphes $Q_{2}$ et $Q_{1} \oplus Q_{0}$ sont canoniquement $F_{2}$-feuilletés, mais ces fibrés ne sont en général pas isomorphes comme fibrés vectoriels $F_{2}$-feuilletés.

Soient maintenant $\nabla$ une connexion sur $Q_{0}$ et $\pi_{1}^{\prime}: T M \longrightarrow F_{1}$ une scission de la suite exacte de fibrés vectoriels

$$
0 \longrightarrow F_{1} \stackrel{i_{1}}{\longrightarrow} T M \stackrel{\pi_{1}}{\longrightarrow} Q_{1} \longrightarrow 0 \text {. }
$$

La torsion de $\nabla$ par rapport à $\pi_{1}^{\prime}$ est la 2-forme $T=T_{\pi_{1}^{\prime}}$ sur $M$ à valeurs dans $Q_{0}$ donnée par la dérivée extérieure covariante de $\pi_{0}^{\prime}=\pi_{0}^{\circ} \pi_{1}^{\prime}$ : $T M \longrightarrow F_{1} \longrightarrow Q_{0}$ (par rapport à $\nabla$ ), où $\pi_{0}$ est la projection canonique, c'est-à-dire

$T(X, Y)=\nabla_{X} \pi_{0}^{\prime}(Y)-\nabla_{Y} \pi_{0}^{\prime}(X)-\pi_{0}^{\prime}[X, Y]$ pour tout $X, Y \in \Gamma(T M)$. Par conséquent, on a donc

$$
T(X, Y)=\nabla_{X} \pi_{0}(Y)-\nabla_{Y} \pi_{0}(X)-\pi_{0}[X, Y] \text { pour tout } X, Y \in \Gamma\left(F_{1}\right) .
$$

Ainsi, pour $F_{1}=T M$, cette notion coïncide avec la notion habituelle.

D'autre part, par un raisonnement analogue à celui utilisé par CorderoMasa dans [3], nous obtenons alors le résultat suivant.

Proposition 4.1. i) Une connexion $\nabla$ sur $Q_{0}$ est adaptée (à la connexion partielle de Bott sur $\left.Q_{0}\right)$ si et seulement si $i_{1}{ }^{*}(i(X) T)=0$ pour tout $X$ $\in \Gamma\left(F_{2}\right)$, où $T=T_{\pi_{1}{ }^{\prime}}$ est la torsion de $\nabla$ par rapport à une scission $\pi_{1}^{\prime}$ quelconque de la suite exacte précédente, et où $i(X)$ est l'opérateur substitution.

ii) Pour que les fibrés $Q_{2}$ et $Q_{1} \oplus Q_{0}$ soient isomorphes comme fibrés vectoriels $F_{2}$-feuilletés il faut et il suffit qu'il existe une scission $\pi_{1}^{\prime}$ de la suite exacte précédente telle que $i(X) T=0$ pour tout $X \in \Gamma\left(F_{2}\right)$, où $T=T_{\pi_{1}{ }^{\prime}}$ est la torsion d'une connexion $F_{2}$-adaptée $\nabla$ sur $Q_{0}$ quelconque.

iii) Il existe un feuilletage $F^{\prime}$ de codimension $d=q_{2}-q_{1} \geqq 0$ sur $M$ tel que $F_{2}=F_{1} \cap F^{\prime}$ si et seulement si l'on peut choisir une connexion $\nabla$ sans torsion sur $Q_{0}\left(\right.$ i.e. $T=T_{\pi_{1}^{\prime}}=0$ ) par rapport à quelque scission $\pi_{1}^{\prime}$ de la suite exacte précédente.

On en déduit le

Corollaire 4.2. S'il existe un feuilletage $F^{\prime}$ de codimension $d=q_{2}-q_{1}$ 
sur $M$ tel que $F_{2}=F_{1} \cap F^{\prime}$, alors $Q_{2}$ et $Q_{1} \oplus Q_{0}$ sont isomorphes comme fibrés vectoriels $F_{2}$-feuilletés. L'implication réciproque est aussi valable pour $q_{1}=$ 1.

Remarque. Si $F$ est un feuilletage de codimension $q$ sur $M$ considéré comme un sous-feuilletage des trois façons différentes, on a (i) $F^{\prime}=T M$ pour $F_{1}=F_{2}=F$ et $q_{1}=q_{2}=q$; (ii) $\quad F^{\prime}=F$ pour $F_{1}=T M, F_{2}=F, q_{1}=0$ et $q_{2}$ $=q$; (iii) pour $F_{1}=F, F_{2}=0, q_{1}=q$ et $q_{2}=n$, on peut seulement affirmer que $Q_{2}=T M$ et $Q_{1} \oplus Q_{0}=(T M / F) \oplus F$ sont isomorphes comme fibrés vectoriels 0 -feuilletés.

Pour interpréter géométriquement certaines classes caractéristiques d'un sous-feuilletage, nous considérons le

Lemme 4.3. Si $Q_{2}$ et $Q_{1} \oplus Q_{0}$ sont isomorphes comme fibrés vectoriels $F_{2}$-feuilletés, il existe une connexion $F_{2}$-adaptée $D$ sur $Q_{0}$ de courbure $R$ telle que

$$
R(X, Y)=0 \text { pour tout } X \in \Gamma\left(F_{2}\right) \text { et tout } Y \in \Gamma\left(\operatorname{Ker} \pi_{1}{ }^{\prime} \cong \Gamma\left(Q_{1}\right),\right.
$$

où $\pi_{1}^{\prime}$ est la scission de $0 \longrightarrow F_{1} \longrightarrow T M \longrightarrow Q_{1} \longrightarrow 0$ donnée dans la Proposition 4.1, partie ii).

Démonstration. Soit g une métrique riemannienne sur $M$ telle que $\operatorname{Ker} \pi_{1}^{\prime} \cong Q_{1}$ soit le fibré complément orthogonal de $F_{1}$ dans $T M$. En utilisant cette métrique, le fibré $Q_{2}$ (resp. $Q_{0}$ ) est isomorphe au fibré complément orthogonal de $F_{2}$ dans $T M$ (resp. dans $F_{1}$ ). Par conséquent,

$$
F_{1} \cong F_{2} \oplus Q_{0}, \quad Q_{2} \cong Q_{0} \oplus Q_{1} \text { et } \quad T M \cong F_{1} \oplus Q_{1} \cong F_{2} \oplus Q_{2} \cong F_{2} \oplus Q_{0} \oplus Q_{1} \text {. }
$$

Considérons maintenant la seconde connexion $\tilde{\nabla}$ de la variété feuilletée riemannienne $\left(M, \mathrm{~g}, F_{1}\right)$ au sens de Vaisman ([13], p.181). Puisque l'appli-

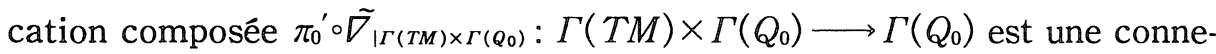
xion $\nabla^{0}$ sur $Q_{0}$, on définit alors une connexion $F_{2}$-adaptée $D$ sur $Q_{0}$ en posant pour tout $X=X_{1}+X_{2} \in \Gamma(T M), X_{1} \in \Gamma\left(F_{2}\right)$ et $X_{2} \in \Gamma\left(Q_{2}\right)$, et tout $Z \in \Gamma\left(Q_{0}\right)$,

$$
\nabla_{X} Z=\pi_{0}\left[X_{1}, Z\right]+\nabla_{X_{2}}^{0} Z .
$$

Compte tenu de la Proposition 4.1, partie ii), on obtient

$$
R(X, Y)=0 \text { pour tout } X \in \Gamma\left(F_{2}\right) \text { et tout } Y \in \Gamma\left(\operatorname{Ker} \pi_{1}{ }^{\prime}\right) \cong \Gamma\left(Q_{1}\right),
$$

$R$ étant la courbure de $\nabla$, d'où le lemme.

On déduit de ce lemme le 
Théorème 4.4. Si $Q_{2}$ et $Q_{1} \oplus Q_{0}$ sont isomorphes comme fibrés vectoriels $F_{2}$-feuilletés, l'homomorphisme caractéristique $\Delta_{*}$ du sous-feuilletage $\left(F_{1}, F_{2}\right)$ se factorise de la manière suivante:

$$
\Delta_{*}: H\left(W O_{I}\right) \stackrel{p_{*}}{\longrightarrow} H\left(W\left(g l\left(q_{1}\right) \oplus g l(d), O\left(q_{1}\right) \times O(d)\right)_{I^{\prime}}\right) \longrightarrow H_{D R}(M),
$$

où $p$ est la projection canonique induite par l'inclusion

$$
I \subset I^{\prime}=I+W\left(g l\left(q_{1}\right)\right) \otimes F^{\left.2\left(q_{1} / 2\right]+d+1\right)} W(g l(d)) .
$$

Ainsi, avec les hypothèses du théorème précédent, les classes de Godbillon-Vey

$$
\Delta *\left[y_{1}^{\prime} \otimes{C_{1}}^{q_{1}-k} C_{1}{ }^{d+k}\right] \text { et } \quad \Delta_{*}\left[y_{1} \wedge y_{1}^{\prime} \otimes C_{1}^{q_{1}-k} C_{1}{ }^{d+k}\right]
$$

de ce sous-feuilletage sont nulles pour $k=\left[q_{1} / 2\right]+1, \cdots, q_{1}$.

On en déduit le

Corollaire 4.5. Pour que les structures $F_{2}$-feuilletées de $Q_{2}$ et $Q_{1} \oplus Q_{0}$ soient incompatibles il suffit que l'une des classes de Godbillon-Vey précédentes soient non nulles.

Dans le cas où $F_{2}=F_{1} \cap F^{\prime}$ pour $F^{\prime}$ de codimension $d=q_{2}-q_{1}$, le fibré vectoriel $F_{2}$-feuilleté $Q_{0} \cong T M / F^{\prime}$ est aussi $F^{\prime}$-feuilleté. Par suite,

Théorème 4.6. S'il existe un feuilletage $F^{\prime}$ de codimension $d=q_{2}-q_{1}$ sur $M$ tel que $F_{2}=F_{1} \cap F^{\prime}$, l'homomorphisme caractéristique $\Delta_{*} d u$ sousfeuilletage $\left(F_{1}, F_{2}\right)$ se factorise comme suit :

$$
\begin{aligned}
\Delta_{*}: & H\left(W O_{I}\right) \stackrel{p_{*}^{\prime}}{\longrightarrow} H\left(W O_{q_{1}}\right) \otimes H\left(W O_{d}\right) \\
& \stackrel{\Delta_{*}\left(Q_{1}\right) \otimes \Delta_{* F^{\prime}}\left(Q_{0}\right)}{\longrightarrow} H_{D R}(M) \otimes H_{D R}(M) \stackrel{\mu}{\longrightarrow} H_{D R}(M),
\end{aligned}
$$

où $\mu$ est la multiplication de $H_{D R}(M)$, où $\Delta_{*}\left(Q_{1}\right)$ (resp. $\Delta_{F^{\prime}}\left(Q_{0}\right)$ ) est l'homomorphisme caractéristique du feuilletage $F_{1}$ (resp. du feuilletage $F^{\prime}$ ), et où $p^{\prime}$ est la projection canonique induite par l'inclusion

$$
I \subset\left(F^{2\left(q_{1}+1\right)} W\left(g l\left(q_{1}\right)\right)\right) \otimes W(g l(d))+W\left(g l\left(q_{1}\right)\right) \otimes F^{2(d+1)} W(g l(d)) .
$$

Pour $0<q_{1}<q_{2}$, le théorème précédent implique que les uniques classes de Godbillon-Vey du sous-feuilletage $\left(F_{1}, F_{2}\right)=\left(F_{1}, F_{1} \cap F^{\prime}\right)$ qui peuvent être non nulles sont les deux classes suivantes:

$$
\Delta *\left[y_{1} \otimes c_{1}^{q_{1}}\right] \text { et } \quad \Delta *\left[y_{1} \wedge y_{1}^{\prime} \otimes c_{1}^{q_{1}} c_{1}^{{ }^{d}}\right] \text {; }
$$


par conséquent, la classe de Godbillon-Vey du feuilletage $F_{2}$ est nulle (cf. aussi Moussu [12]).

On en déduit le

Corollaire 4.7. Si l'une des classes de Godbillon-Vey

$$
\Delta_{*}\left[y_{1}^{\prime} \otimes C_{1}^{q_{1}-k} C_{1}{ }^{d+k}\right] \text { et } \Delta_{*}\left[y_{1} \wedge y_{1}^{\prime} \otimes C_{1}^{q_{1}-k} C_{1}^{{ }^{d+k}}\right], \quad 1 \leqq k \leqq q_{1},
$$

n'est pas nulle, il n'existe aucun feuilletage $F^{\prime}$ de codimension $d=q_{2}-q_{1}$ sur $M$ tel que $F_{2}=F_{1} \cap F^{\prime}$.

Remarque. Il est clair que toutes les classes de Godbillon-Vey de ( $F_{1}$, $F_{2}$ ) sont nulles dans les deux cas suivants :

(i) le fibré des repères transverses à ce sous-feuilletage admet une connexion basique somme;

(ii) le fibré normal de ce sous-feuilletage est orientable (i.e. $Q_{1}$ et $Q_{0}$ sont des fibrés vectoriels orientables) et la structure $\left(F_{1}, F_{2}\right)$-feuilletée de $P=$ $L\left(Q_{1}\right)+L\left(Q_{0}\right)$ est induite par une structure $\left(F_{1}, F_{2}\right)$-feuilletée d'une $S L\left(q_{1}\right) \times$ $S L(d)$-réduction $P^{\prime}=P_{1}^{\prime}+P_{2}^{\prime}$ de $P$.

\section{$\S 5$. Classes de Godbillon-Vey d'un Sous-feuilletage}

Dans ce paragraphe, en reprenant les notations du $\S 4$, nous construisons les classes de Godbillon-Vey d'un sous-feuilletage de deux façons différentes, l'une d'elles est basée sur les techniques de Godbillon-Vey [5], l'autre utilise l'homomorphisme caractéristique du sous-feuilletage.

a) Construction de ces classes par les techniques de Godbillon-Vey.

Soit $0 \longrightarrow Q_{0} \stackrel{i}{\longrightarrow} Q_{2} \stackrel{\pi}{\longrightarrow} Q_{1} \longrightarrow 0$ la suite exacte de fibrés vectoriels, canoniquement associée à $\left(F_{1}, F_{2}\right)$. Soint $Q_{i}{ }^{*}, i=0,1,2$, les fibrés vectoriels duals de $Q_{i}$; on considère $Q_{0}{ }^{*}$ comme un sous-fibré vectoriel de $Q_{2}{ }^{*}$, en utilisant une scission $p^{*}: \mathrm{Q}_{0}{ }^{*} \longrightarrow \mathrm{Q}_{2}{ }^{*}$ de la suite exacte de fibrés vectoriels

$$
0 \longrightarrow Q_{1}{ }^{*} \stackrel{\pi^{*}}{\longrightarrow} Q_{2}{ }^{*} \stackrel{i^{*}}{\longrightarrow} Q_{0}{ }^{*} \longrightarrow 0 .
$$

Par conséquent, on a donc $\wedge Q_{1}{ }^{*} \otimes \wedge Q_{0}{ }^{*} \cong \wedge Q_{2}{ }^{*} \subset \wedge T M^{*}$.

Supposons maintenant que $\left(F_{1}, F_{2}\right)$ soit un sous-feuilletage à fibré normal orientable (c'est $Q_{1}$ et $Q_{0}$ sont des fibrés vectoriels orientables). Soient $\gamma_{1}$ et $\gamma_{2}$ deux formes volumes de $Q_{1}$ et $Q_{0}$ respectivement; en 
particulier, $\gamma=\gamma_{1} \Lambda \gamma_{2}$ est une forme volume de $Q_{2}$. Alors il existe un recouvrement ouvert $R=\{U\}$ de $M$ et un repère mobile $\left\{\omega_{2}\right\}, 1 \leqq i \leqq q_{2}$, sur $U$ pour $Q_{2}{ }^{*} \cong Q_{1}{ }^{*} \oplus Q_{0}{ }^{*} \subset T M^{*}$ tel que $\left\{\omega_{u}\right\}, 1 \leqq u \leqq q_{1}$ (resp. $\left\{\omega_{a}\right\}, q_{1}+1 \leqq a \leqq$ $q_{2}$ \} soit un repère mobile sur $U$ pour $Q_{1}{ }^{*}$ (resp. pour $Q_{0}{ }^{*}$ ), et tel que, sur chaque $U \in \mathscr{R}$, on ait

$$
\gamma_{1}=\omega_{1} \wedge \cdots \wedge \omega_{q_{1}} \text { et } \quad \gamma_{2}=\omega_{q_{1}+1} \wedge \cdots \wedge \omega_{q_{2}} .
$$

Par le théorème de Frobenius, il existe des 1 -formes locales $\alpha_{u v}, \alpha_{a v}$ et $\alpha_{a b}$, $1 \leqq u, v \leqq q_{1}, q_{1}+1 \leqq a, b \leqq q_{2}$, sur $M$ telles que $d \omega_{u}=\sum_{v=1}^{q_{1}} \alpha_{u v} \wedge \omega_{v}$ et $d \omega_{a}=$ $\sum_{v=1}^{q_{1}} \alpha_{a v} \wedge \omega_{v}+\sum_{b=q_{1}+1}^{q_{2}} \alpha_{a b} \wedge \omega_{b}$. Ainsi, par différentiation de ces relations, on obtient, sur chaque $U \in \mathscr{R}$,

$$
\begin{aligned}
d \gamma_{1} & =\tau_{1}{ }^{U} \Lambda \gamma_{1} \quad \text { et } \quad d \gamma_{2}-\tau_{2}{ }^{U} \Lambda \gamma_{2}=\sum_{v=1}^{q_{1}} \omega_{v} \wedge \phi_{v} \in F^{1} \Omega(U) \\
& =\Gamma\left(U, \underline{Q}_{1}{ }^{*} \cdot \Omega_{M}\right) \subset \Omega(U),
\end{aligned}
$$

où $\tau_{1}^{U}=\sum_{u=1}^{q_{1}} \alpha_{u u}, \quad \tau_{2}{ }^{U}=\sum_{a=q_{1}+1}^{q_{2}} \alpha_{a a}$ et $\phi_{v}=\sum_{a=q_{1}+1}^{q_{2}}(-1)^{a-q_{1}} \alpha_{a v} \wedge \omega_{q_{1}+1} \wedge \cdots \wedge \widehat{\omega}_{a} \wedge$ $\cdots \wedge \omega_{q_{2}}$, et où $\underline{Q}_{1}{ }^{*}$ désigne le faisceau de 1-formes locales annulant $F_{1}$.

D'autre part, considérons une partition différentiable de l'unité $\left\{\lambda^{U}\right\}$ subordonnée au recouvrement $\mathcal{R}$. On définit alors deux 1 -formes $\tau_{1}$ et $\tau_{2}$ sur $M$ en posant

$$
\tau_{i}=\sum_{U \in \mathbb{R}} \lambda^{U} \cdot \tau_{i}{ }^{U} \quad \text { pour } \quad i=1,2
$$

Il en résulte que

$$
d \gamma_{1}=\tau_{1} \wedge \gamma_{1} \quad \text { et } \quad d \gamma_{2}-\tau_{2} \wedge \gamma_{2} \in F^{1} \Omega(M)=\Gamma\left(M, \underline{Q}^{*} \cdot \Omega_{M}\right) \subset \Omega(M) .
$$

En particulier, on a donc $d \gamma=\tau \wedge \gamma$ avec $\tau=\tau_{1}+\tau_{2}$.

Nous obtenons alors le résultat suivant.

Lemme 5.1. Les formes $\tau_{1} \wedge\left(d \tau_{1}\right)^{q_{1}}, \tau_{2} \wedge\left(d \tau_{1}\right)^{j} \wedge\left(d \tau_{2}\right)^{q_{2}-j}$ et $\tau_{1} \wedge \tau_{2} \wedge\left(d \tau_{1}\right)^{\prime} \wedge\left(d \tau_{2}\right)^{q_{2}-\rho}, 0 \leqq j \leqq q_{1}$, sur $M$ sont fermées, et leurs classes de cohomologie dans $H_{D R}(M)$ ne dépendent que du sous-feuilletage $\left(F_{1}, F_{2}\right)$.

On peut généraliser la construction précédente au cas général d'un sous-feuilletage $\left(F_{1}, F_{2}\right)$ dont le fibré normal $Q_{1} \oplus Q_{0}$ n'est pas nécessairement orientable comme suit. Soit $\mathcal{R}=\{U\}$ un recouvrement ouvert de $M$ tel qu'il existe un repère mobile normé $\gamma_{1}{ }^{U}$ (resp. $\gamma_{2}{ }^{U}$ ) sur $U$ pour $\wedge^{q_{1}} Q_{1}{ }^{*}$ 
(resp. pour $\wedge^{d} Q_{0}{ }^{*}$ ) par rapport à une métrique riemannienne fixe sur $\wedge^{q_{1}} Q_{1}{ }^{*}$ (resp. sur $\wedge^{d} Q_{0}{ }^{*}$ ). En particulier, $\gamma^{U}=\gamma_{1}{ }^{U} \wedge \gamma_{2}{ }^{U}$ est un repère mobile normé sur $U$ pour $\wedge^{q_{2}} Q_{2}{ }^{*} \cong \wedge^{q_{1}} Q_{1}{ }^{*} \otimes \wedge^{d} Q_{0}{ }^{*}$ par rapport à la métrique riemannienne induite. Alors, par une technique similaire à celle utilisée dans le cas précédent, on construit deux 1 -formes $\tau_{1}$ et $\tau_{2}$ sur $M$ (entièrement définies) telles que, sur chaque $U \in \mathscr{R}, d \gamma_{1}{ }^{U}=\tau_{1} \wedge \gamma_{1}{ }^{U}$ et $d \gamma_{2}{ }^{U}$ $-\tau_{2} \wedge \gamma_{2}{ }^{U} \in F^{1} \Omega(U)$. Par suite, $d \gamma^{U}=\tau \wedge \gamma^{U}$ avec $\tau=\tau_{1}+\tau_{2}$. Compte tenu du lemme précédent et du fait qu'il existe un revêtement à quatre feuillets $f: M^{\prime} \longrightarrow M$ tel que $f^{-1}\left(F_{1}, F_{2}\right)=\left(f^{-1}\left(F_{1}\right), f^{-1}\left(F_{2}\right)\right)$ soit un sous-feuilletage de codimension $\left(q_{1}, q_{2}\right)$ sur $M^{\prime}$ dont le fibré normal est orientable, on obtient le résultat suivant.

Théorème 5.2. Les formes $\tau_{1} \wedge\left(d \tau_{1}\right)^{q_{1}}, \quad \tau_{2} \wedge\left(d \tau_{1}\right)^{j} \wedge\left(d \tau_{2}\right)^{q_{2}-j}$ et $\tau_{1} \wedge \tau_{2} \wedge\left(d \tau_{1}\right)^{j} \wedge\left(d \tau_{2}\right)^{q_{2}-j}, 0 \leqq j \leqq q_{1}$, sur $M$ sont fermées, et leurs classes de cohomologie dans $H_{D R}(M)$ ne dépendent que du sous-feuilletage $\left(F_{1}, F_{2}\right)$.

De telles classes de cohomologie dans $H_{D R}(M)$ seront appelées les classes de Godbillon-Vey du sous-feuilletage $\left(F_{1}, F_{2}\right)$.

Remarques. 1) Il est clair que $\left[\tau_{1} \wedge\left(d \tau_{1}\right)^{q_{1}}\right] \in H_{D R}^{2 q_{1}+1}(M)$ est la classe de Godbillon-Vey du feuilletage $F_{1}$ et que (pour $q_{1}<q_{2}$ )

$$
\left[\tau \wedge(d \tau)^{q_{2}}\right]=\sum_{j=0}^{q_{1}}\left(\begin{array}{c}
q_{2}+1 \\
j
\end{array}\right)\left[\tau_{2} \wedge\left(d \tau_{1}\right)^{j} \wedge\left(d \tau_{2}\right)^{q_{2}-j}\right] \in H_{D R}^{2 q_{2}+1}(M)
$$

est la classe de Godbillon-Vey du feuilletage $F_{2}$.

2) Du Théorème 5.2 on déduit immédiatement que l'homomorphisme $f^{*}: H_{D R}(M) \longrightarrow H_{D R}(N)$ induit en cohomologie par une application différentiable transverse $f: N \longrightarrow M$ à $\left(F_{1}, F_{2}\right)$, transforme chaque classe de Godbillon-Vey de $\left(F_{1}, F_{2}\right)$ en la classe correspondante de Godbillon-Vey du sous-feuilletage $f^{-1}\left(F_{1}, F_{2}\right)$ sur la variēté $N$. En particulier, les classes de Godbillon-Vey d'un sous-feuilletage ne dépendent que de la classe d'homotopie de ce sous-feuilletage.

b) Construction des classes de Godbillon-Vey en utilisant l'homomorphisme caractéristique.

Soit $P=L\left(Q_{1}{ }^{*}\right)+L\left(Q_{0}{ }^{*}\right)$ le fibré principal $\left(F_{1}, F_{2}\right)$-feuilleté des $G L\left(q_{1}\right)$ $\times G L(d)$-repères de $Q_{1}{ }^{*} \oplus Q_{0}{ }^{*}$. Alors le fibré principal $\left(F_{1}, F_{2}\right)$-feuilleté $P^{\prime}$ $=L\left(\wedge^{q_{1}} Q_{1}{ }^{*}\right)+L\left(\wedge^{d} Q_{0}{ }^{*}\right)$ des $G L(1) \times G L(1)$-repères de $\wedge^{q_{1}} Q_{1}{ }^{*} \oplus \wedge^{d} Q_{0}{ }^{*}$ est associé à $P$ par l'homomorphisme de groupes de Lie $\rho=$ (dét, dét): $G L\left(q_{1}\right)$ 
$\times G L(d) \longrightarrow G L(1) \times G L(1) . \quad$ Par suite, l'homomorphisme caractéristique généralisé $U_{*}^{\prime}$ de $P^{\prime}$ est donné par la composition

$$
\Delta^{\prime}: H\left(W(g l(1) \oplus g l(1), O(1) \times O(1))_{I}\right) \stackrel{w(d \rho)^{*}}{\longrightarrow} H\left(W O_{I}\right)_{I} \stackrel{\Delta_{*}}{\longrightarrow} H_{D R}(M),
$$

où $\Delta_{*}$ désigne l'homomorphisme caractéristique généralisé de $P$.

Soit maintenant $\nabla^{1 *} \oplus D^{2 *}$ une connexion adaptée somme sur le fibré vectoriel $\left(F_{1}, F_{2}\right)$-feuilleté $\wedge^{q_{1}} Q_{1}{ }^{*} \oplus \wedge^{d} Q_{0}{ }^{*}$. Considérons des métriques riemanniennes sur $\wedge^{q_{1}} Q_{1}{ }^{*}$ et $\wedge^{d} Q_{0}{ }^{*}$ respectivement. Soit $\gamma_{1}$ (resp. $\gamma_{2}$ ) un repère mobile normé (de norme 1) pour $\wedge^{q_{1}} Q_{1}{ }^{*}$ (resp. pour $\wedge^{d} Q_{0}{ }^{*}$ ) sur un ouvert $U$ de $M$ au-dessus duquel $\wedge^{q_{1}} Q_{1}{ }^{*}$ (resp. $\wedge^{d} Q_{0}{ }^{*}$ ) est trivial. Alors, par un raisonnement analogue à celui par lequel on a prouvé le Théorème 7.20 dans [8], on en déduit le

Théorème 5.3. Les classes de Godbillon-Vey $\Delta_{*}\left[y_{1} \otimes c_{1}{ }^{q_{1}}\right]$, $\Delta_{*}\left[y_{1}^{\prime} \otimes c_{1}{ }^{j} c_{1}{ }^{q_{2}-\jmath}\right]$ et $\Delta_{*}\left[y_{1} \wedge y_{1}^{\prime} \otimes c_{1}{ }^{j} c_{1}{ }^{q_{2}-\jmath}\right], 0 \leqq j \leqq q_{1}$, sont réalisées respectivement par les formes fermées $\sigma_{1} \wedge\left(d \sigma_{1}\right)^{q_{1}}, \sigma_{2} \wedge\left(d^{\prime} \sigma_{1}\right)^{j} \wedge\left(d \sigma_{2}\right)^{q_{2}-\rho}$ et $\sigma_{1} \wedge \sigma_{2} \wedge\left(d \sigma_{1}\right)^{\jmath} \wedge\left(d \sigma_{2}\right)^{q_{2}-\jmath}, 0 \leqq j \leqq q_{1}$, sur $M$, où les 1 -formes $\sigma_{1}$ et $\sigma_{2}$ sur $M$ (entièrement définies) sont déterminées (sur $U$ ) par les formules

$$
\nabla_{X}^{1 *} \gamma_{1}=2 \pi \sigma_{1}(X) \gamma_{1} \text { et } \nabla_{X}^{2 *} \gamma_{2}=2 \pi \sigma_{2}(X) \gamma_{2} \text { pour } X \in \Gamma(T M) .
$$

Par conséquent, pour chaque $i=1,2,2 \pi \sigma_{2}$ est la forme de la connexion $\nabla^{2 *}, 2 \pi d \sigma_{2}$ sa forme de courbure, associée au repère mobile normé $\gamma_{2}$ sur $U$.

Remarque. $\mathrm{Si}\left(F_{1}, F_{2}\right)$ est un sous-feuilletage à fibré normal orientable, il est clair que l'on peut choisir les repères mobiles précédents de telle sorte que $\gamma_{1}$ et $\gamma_{2}$ soient des formes volumes de $Q_{1}$ et $Q_{0}$ respectivement.

c) Relation entre les classes de Godbillon-Vey construites par les voies a) et b).

Démontrons d'abord le résultat suivant.

Lemme 5.4. Il existe des connexions adaptées $\nabla^{1}, \nabla$ et $\nabla^{2}$ sur $Q_{1}, Q_{2}$ et $Q_{0}$ compatibles avec les homomorphismes canoniques $\pi: Q_{2} \longrightarrow Q_{1}$ et $i: Q_{0} \longrightarrow Q_{2}$ telles que $\nabla^{1}$ et $\nabla$ soient des connexions sans torsion.

Démonstration. Soit $\mathrm{g}$ une métrique riemannienne sur $M$. On identifie alors $Q_{2}, i=1,2$ (resp. $Q_{0}$ ) avec le fibré complément orthogonal de $F_{2}$ dans $T M$ (resp. de $F_{2}$ dans $F_{1}$ ). Soit $\tilde{\nabla}$ la seconde connexion de la variété feuilletée riemannienne $\left(M, \mathrm{~g}, F_{1}\right)$. Alors l'application 


$$
\nabla^{1}=\pi_{1} \circ \tilde{\nabla}_{\mid \Gamma(T M) \times \Gamma\left(Q_{1}\right)}: \Gamma(T M) \times \Gamma\left(Q_{1}\right) \longrightarrow \Gamma\left(Q_{1}\right)
$$

est une connexion $F_{1}$-adaptée sur $Q_{1}$ et sans torsion. D'autre part, en

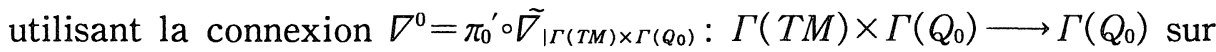
$Q_{0}$, on définit une connexion $F_{2}$-adaptée $\nabla^{2}$ sur $Q_{0}$ de torsion $T_{2}$ en posant, pour tout $X=X_{1}+X_{2} \in \Gamma(T M), X_{1} \in \Gamma\left(F_{2}\right)$ et $X_{2} \in \Gamma\left(Q_{2}\right)$, et tout $Z \in \Gamma\left(Q_{0}\right)$, $\nabla_{X}^{2} Z=\pi_{0}\left[X_{1}, Z\right]+\nabla_{X_{2}}^{0} Z$.

On peut maintenant considérer la connexion $\nabla^{\prime}$ sur $Q_{2} \cong Q_{0} \oplus Q_{1}$ donnée, pour tout $X \in \Gamma(T M)$, par la formule

$$
\begin{aligned}
& \nabla_{X}^{\prime} Z=\left(\nabla_{X}^{2} Z_{0}-\frac{1}{2} T_{2}\left(X, Z_{1}\right)\right)+\nabla_{X}^{1} Z_{1}, \quad Z=Z_{0}+Z_{1} \in \Gamma\left(Q_{2}\right), \\
& Z_{0} \in \Gamma\left(Q_{0}\right), \quad Z_{1} \in \Gamma\left(Q_{1}\right) .
\end{aligned}
$$

On définit alors une connexion $F_{2}$-adaptée $\nabla$ sur $Q_{2}$ et sans torsion en posant, pour tout $X=X_{1}+X_{2} \in \Gamma(T M), X_{1} \in \Gamma\left(F_{2}\right)$ et $X_{2} \in \Gamma\left(Q_{2}\right)$, et tout $Z \in \Gamma\left(Q_{2}\right)$,

$$
\nabla_{X} Z=\pi_{2}\left[X_{1}, Z\right]+\nabla_{X_{2}}^{\prime} Z \text {. }
$$

Les connexions $\nabla^{1}, \nabla$ et $\nabla^{2}$ sont évidemment compatibles avec les homomorphismes canoniques $\pi$ et $i$. Ceci achève la démonstration du lemme.

On dira, d'un triplet $\left(\nabla^{1}, \nabla, \nabla^{2}\right)$ de connexions adaptées qu'il est adapté au sous-feuilletage $\left(F_{1}, F_{2}\right)$, si ces connexions vérifient les propriétés précédentes (cf. aussi Cordero-Masa [3]).

Soit $\left(\nabla^{1}, \nabla, \nabla^{2}\right)$ un triplet adapté de connexions adaptées. Considérons le triplet adapté $\left(\nabla^{1 *}, \nabla^{*}, \nabla^{2 *}\right)$ de connexions adaptées sur $Q_{1}{ }^{*}, Q_{2}{ }^{*}$ et $Q_{0}{ }^{*}$ induit $\operatorname{par}\left(\nabla^{1}, \nabla, \nabla^{2}\right)$. Soit $U$ un ouvert de $M$ au-dessus duquel $Q_{1}{ }^{*}, Q_{2}{ }^{*}$ et $Q_{0}{ }^{*}$ sont triviaux, et soit $\left\{\omega_{i}\right\}, 1 \leqq i \leqq q_{2}$, un repère mobile sur $U$ pour $Q_{2}{ }^{*} \subset$ $T M^{*}$ adapté (au sens de Cordero-Masa [3]), cela signifie que $\left\{\omega_{u}\right\}, 1 \leqq u \leqq q_{1}$ (resp. $\left\{i^{*} \omega_{a}\right\}, q_{1}+1 \leqq a \leqq q_{2}$ ) est un repère mobile sur $U$ pour $Q_{1}{ }^{*} \subset Q_{2}{ }^{*}$ (resp. pour $\left.Q_{0}{ }^{*}\right)$. Alors les matrices $\left(\theta_{u v}^{1}\right),\left(\theta_{i j}\right)$ et $\left(\theta_{a b}^{2}\right)$ des connexions $\nabla^{1 *}, \nabla^{*}$ et $\nabla^{2 *}$ associées au repère mobile adapté $\left\{\omega_{i}\right\}$ sur $U$ vérifient respectivement $\theta_{u v}^{1}=\theta_{u v}, \theta_{u b}=0$ et $\theta_{a b}^{2}=\theta_{a b}$ pour $1 \leqq u, v \leqq q_{1}$ et $q_{1}+1 \leqq a, b \leqq q_{2}$. Par conséquent, les connexions adaptées $\nabla^{1 *}, \nabla^{2 *}$ et $\nabla^{*}$ sur $\wedge^{q_{1}} Q_{1}{ }^{*}, \wedge^{d} Q_{0}{ }^{*}$ et $\wedge{ }^{q_{2}} Q_{2}{ }^{*}$ induites par les connexions précédentes sont données respectivement, pour tout $X \in \Gamma(T M)$, par les formules

$$
\nabla_{X}^{1 *} \gamma_{1}=\tau_{1}(X) \gamma_{1}, \quad \nabla_{X}^{2 *} i^{*} \gamma_{2}=\tau_{2}(X) i^{*} \gamma_{2} \text { et } \nabla_{X}^{*} \gamma=\tau(X) \gamma,
$$


oũ $\gamma_{1}=\omega_{1} \wedge \cdots \wedge \omega_{q_{1}}, \gamma_{2}=\omega_{q_{1}+1} \wedge \cdots \wedge \omega_{q_{2}}, \gamma=\gamma_{1} \wedge \gamma_{2}, \tau_{1}=\sum_{u=1}^{q_{1}} \theta_{u u}, \tau_{2}=\sum_{a=q_{1}+1}^{q_{2}} \theta_{a a}$ et $\tau=\tau_{1}+\tau_{2}$. Il s'ensuit que $\wedge^{q_{1}} Q_{1}{ }^{*} \otimes \wedge^{d} Q_{0}{ }^{*}$ et $\wedge^{q_{2}} Q_{2}{ }^{*}$ sont isomorphes comme fibrés vectoriels $F_{2}$-feuilletés.

D'autre part, puisque $\nabla^{1}$ et $\nabla$ sont des connexions sans torsion, on a alors

$$
\begin{gathered}
d \omega_{u}=\sum_{v=1}^{q_{1}} \theta_{u v} \wedge \omega_{v} \text { et } d \omega_{a}=\sum_{v=1}^{q_{1}} \theta_{a v} \wedge \omega_{v}+\sum_{b=q_{1}+1}^{q_{2}} \theta_{a b} \wedge \omega_{b} \\
\text { pour } 1 \leqq u \leqq q_{1} \text { et } \quad q_{1}+1 \leqq a \leqq q_{2} .
\end{gathered}
$$

Par différentiation de ces relations, on obtient donc

$$
d \gamma_{1}=\tau_{1} \Lambda \gamma_{1}, \quad d \gamma_{2}=\sum_{v=1}^{q_{1}} \omega_{v} \Lambda \phi_{v}+\tau_{2} \wedge \gamma_{2} \text { et } \quad d \gamma=\tau \wedge \gamma,
$$

où $\phi_{v}=\sum_{a=q_{1}+1}^{q_{2}}(-1)^{a-q_{1}} \theta_{a v} \wedge \omega_{q_{1}+1} \wedge \cdots \wedge \bar{\omega}_{a} \wedge \cdots \wedge \omega_{q_{2}}$.

Supposons maintenant que $\gamma_{1}$ (resp. $i^{*} \gamma_{2}$ ) soit un repère mobile normé sur $U$ pour $\wedge^{q_{1}} Q_{1}{ }^{*}$ (resp. pour $\wedge^{d} Q_{0}{ }^{*}$ ) par rapport à une métrique riemannienne fixe sur $\wedge^{q_{1}} Q_{1}{ }^{*}$ (resp. sur $\wedge^{d} Q_{0}{ }^{*}$ ). Il en résulte que $\tau_{1}, \tau_{2}$ et $\tau=\tau_{1}+\tau_{2}$ sont des 1-formes sur $M$ (entièrement définies) satisfaisant, sur chaque ouvert $U \subset M$ de la trivialisation locale,

$$
d \gamma_{1}=\tau_{1} \wedge \gamma_{1} \quad \text { et } \quad d \gamma_{2}-\tau_{2} \wedge \gamma_{2} \in F^{1} \Omega(U) .
$$

De plus, on a donc

$$
\tau_{i}=2 \pi \sigma_{2}, \quad i=1,2, \quad \text { et } \quad \tau=\tau_{1}+\tau_{1}=2 \pi \sigma_{1}+2 \pi \sigma_{1},
$$

où les $\sigma_{\imath}$ sont les 1 -formes sur $M$ données dans le Théorème 5.3. On a ainsi démontré le

Théorème 5.5. Les classes de Godbillon-Vey construites dans les Théorèmes 5.2 et 5.3 vérifient les relations suivantes:

(i) $\left[\tau_{1} \wedge\left(d \tau_{1}\right)^{q_{1}}\right]=(2 \pi)^{q_{1}+1}\left[\sigma_{1} \wedge\left(d \sigma_{1}\right)^{q_{1}}\right] \in H_{D R}^{2 q_{1}+1}(M)$;

(ii) $\left[\tau_{2} \wedge\left(d \tau_{1}\right)^{\jmath} \wedge\left(d \tau_{2}\right)^{q_{2}-\jmath}\right]=(2 \pi)^{q_{2}+1}\left[\sigma_{2} \wedge\left(d \sigma_{1}\right)^{\jmath} \wedge\left(d \sigma_{2}\right)^{q_{2}-\jmath}\right] \in H_{D R}^{2 q_{2}+1}(M), 0 \leqq j$ $\leqq q_{1}$;

(iii) $\left[\tau_{1} \wedge \tau_{2} \wedge\left(d \tau_{1}\right)^{\jmath} \wedge\left(d \tau_{2}\right)^{q_{2}-\jmath}\right]=(2 \pi)^{q_{2}+2}\left[\sigma_{1} \wedge \sigma_{2} \wedge\left(d \sigma_{1}\right)^{\jmath} \wedge\left(d \sigma_{2}\right)^{q_{2}-\jmath}\right] \in H_{D R}^{2 q_{2}+2}$ $(M), 0 \leqq j \leqq q_{1}$.

\section{§6. Quelques Exemples}

Pour appliquer les résultats des paragraphes précédents, nous donnons 
dans ce paragraphe quelques exemples de sous-feuilletages localement homogènes ayant des classes de Godbillon-Vey non nulles.

Soient $H \subset G_{2} \subset G_{1} \subset \bar{G}$ des groupes de Lie, $h \subset g_{2} \subset g_{1} \subset \bar{g}$ leurs algèbres de Lie. Supposons que $H$ soit fermé dans $\bar{G}$. Soit $\Gamma \subset \bar{G}$ un sous-groupe discret opérant de manière proprement discontinue et sans points fixes sur $\bar{G} / H$. Considérons le sous-feuilletage localement homogène $\left(F_{1}, F_{2}\right)=\left(F_{G_{1}}\right.$, $\left.F_{G_{2}}\right)$ de codimension $\left(q_{1}, q_{2}\right)=\left(\operatorname{dim} \bar{g} / g_{1}, \operatorname{dim} \bar{g} / g_{2}\right)$ sur la variété localement homogène $M=\Gamma \backslash \bar{G} / H$ de dimension $n=\operatorname{dim} \bar{g} / h$ induit par le sousfeuilletage sur $\bar{G}$, déterminé par les orbites des opérations à droite de $G_{1}$ et $G_{2}$. Soit $Q_{1} \oplus Q_{0}$ (resp. $\left.Q_{2}\right)$ le fibré normal de $\left(F_{1}, F_{2}\right)$ (resp. de $\left.F_{2}\right)$. On a alors $d=q_{2}-q_{1}=\operatorname{dim} g_{1} / g_{2}, Q_{i} \cong \Gamma \backslash \bar{G} \times_{H} \bar{g} / g_{i}, i=1$, 2, et $Q_{0} \cong \Gamma \backslash \bar{G} \times_{H} g_{1} / g_{2}$.

Exemple 1. Pour $d \geqq 1$, prenons $\bar{G}=S L(d+2), G_{1}=S L(d+2,1)_{0}, G_{2}=$ $S L(d+2,2)_{0}, H=S O(d)$ et $\Gamma$ un sous-groupe discret, uniforme et sans torsion de $S L(d+2)$, où $S L(d+2,1)_{0}$ (resp. $\left.S L(d+2,2)_{0}\right)$ désigne la composante connexe du groupe $S L(d+2,1)$ (resp. du groupe $S L(d+2,2)$ ) des matrices de déterminant 1 de la forme $\left(\begin{array}{l|l}\lambda & * \\ \hline 0 & A\end{array}\right)$ pour $A \in$ $G L(d+1) \quad$ et $\lambda=\operatorname{dét} A^{-1}$ (resp. $\left[\begin{array}{c|c|c}\lambda_{1} & & * \\ \hline & \lambda_{2} & * \\ \hline 0 & 0 & B\end{array}\right)$ pour $B \in G L(d)$ et $\lambda_{1}, \lambda_{2}$ $\in G L(1)$, avec $\left.\lambda_{1}{ }^{-1}=\lambda_{2} \cdot \operatorname{dét} B\right)$. Soit $\left(F_{1}, F_{2}\right)=\left(F_{G_{1}}, F_{G_{2}}\right)$ le sous-feuilletage localement homogène de codimension $\left(q_{1}, q_{2}\right)=(d+1,2 d+1)$ sur $M=$ $\Gamma \backslash S L(d+2) / S O(d)$ dont le fibré normal $Q_{1} \oplus Q_{0}$ est orientable. Pour appliquer le Lemme 5.1, considérons la scission $p^{*}: Q_{0}{ }^{*} \longrightarrow Q_{2}{ }^{*}$ de 0 $\longrightarrow Q_{1}{ }^{*} \longrightarrow Q_{2}{ }^{*} \longrightarrow Q_{0}{ }^{*} \longrightarrow 0$ induite par la scission $G L(d+1)$-équivariante $\theta: \bar{g} \longrightarrow g_{1}$ de la suite exacte $0 \longrightarrow g_{1} \longrightarrow \bar{g} \longrightarrow \bar{g} / g_{1} \longrightarrow 0$, définie par la formule

$$
\theta\left(\left(\begin{array}{c|c}
\lambda & * \\
\hline * & A
\end{array}\right)\right)=\left(\begin{array}{l|l}
\lambda & * \\
\hline 0 & A
\end{array}\right) .
$$

D'autre part, soit $\left\{x_{i j}^{*}\right\}, 1 \leqq i, j \leqq d+2$, la base duale de la base canonique $\left\{x_{i j}\right\}$ de $g l(d+2)$. On désigne par $\omega_{i j}$ la restriction de $x_{i j}^{*}$ à $s l(d+2)$. On vérifie alors que $\left\{\omega_{i j}\right\} \cup\left\{\omega_{k k}\right\}, 1 \leqq i, j \leqq d+2, i \neq j, 1 \leqq k \leqq d+1$, est la base 
duale de la base canonique de $s l(d+2)$ et que $\sum_{i=1}^{m} \omega_{i i}=0$, où $m=d+2$. De plus, une base de $\left(\bar{g} / g_{1}\right)^{*} \subset\left(\bar{g} / g_{2}\right)^{*}$ (resp. de $\left.\left(g_{1} / g_{2}\right)^{*} \stackrel{\theta^{*}}{\longrightarrow}\left(\bar{g} / g_{2}\right)^{*}\right)$ est donnée par

$$
\left.\left\{\omega_{i 1}\right\}, 2 \leqq i \leqq m \text { (resp. par }\left\{\omega_{i 2}\right\}, 3 \leqq i \leqq m\right) .
$$

Il s'ensuit que les deux bases précédentes fournissent une base de $\left(\bar{g} / g_{2}\right)^{*} \cong$ $\left(\bar{g} / g_{1}\right)^{*} \oplus\left(g_{1} / g_{2}\right)^{*}$. Notons respectivement $\gamma_{1}^{\prime}$ et $\gamma_{2}^{\prime}$ les bases $\omega_{21} \wedge \cdots \wedge \omega_{m 1}$ et $\omega_{32} \wedge \cdots \wedge \omega_{m 2}$ de $\wedge^{d+1}\left(\bar{g} / g_{1}\right)^{*}$ et $\wedge^{d}\left(g_{1} / g_{2}\right)^{*}$. Du fait que la différentielle de Chevalley-Eilenberg $d_{\wedge}$ sur $\wedge \bar{g}^{*}$ est définie par

$$
d_{\wedge} \omega_{i j}=-\sum_{k=1}^{m} \omega_{i k} \wedge \omega_{k j} \quad \text { pour } \quad 1 \leqq i, \quad j \leqq m=d+2,
$$

on obtient donc

$$
d_{\wedge} \gamma_{1}^{\prime}=\tau_{1}^{\prime} \wedge \gamma_{1}^{\prime} \quad \text { et } \quad d_{\wedge} \gamma_{2}^{\prime}=\sum_{i=3}^{m} \omega_{i 1} \wedge \phi_{2}+\tau_{2}^{\prime} \wedge \gamma_{2}^{\prime},
$$

où $\tau_{1}^{\prime}=m \omega_{11}, \tau_{2}^{\prime}=\omega_{11}+(m-1) \omega_{22}$ et $\phi_{i}=(-1)^{i} \omega_{12} \wedge \omega_{32} \wedge \cdots \wedge \widehat{\omega}_{i 2} \wedge \cdots \wedge \omega_{m 2}$. Puisque $\tau_{1}^{\prime}, \tau_{2}^{\prime}, \gamma_{1}^{\prime}, \gamma_{2}^{\prime} \in\left(\wedge \bar{g}^{*}\right)_{H}$ (sous-algèbre des éléments $H$-basiques de $\left.\wedge \bar{g}^{*}\right)$, ces éléments induisent des formes $\tau_{1}, \tau_{2}, \gamma_{1}$ et $\gamma_{2}$ sur $M=\Gamma \backslash \bar{G} / H$ vérifiant

$$
d \gamma_{1}=\tau_{1} \wedge \gamma_{1} \quad \text { et } \quad d \gamma_{2}-\tau_{2} \wedge \gamma_{2} \in F^{1} \Omega(M) .
$$

De plus, $\gamma_{1}$ (resp. $\gamma_{2}$ ) est une forme volume de $Q_{1}$ (resp. de $Q_{0}$ ).

D'autre part, on déduit de $d_{\wedge} \omega_{i i}=-\sum_{k=1}^{m} \omega_{i k} \wedge \omega_{k i}$ les identités suivantes :

$$
\begin{aligned}
& \left(d_{\wedge} \omega_{11}\right)^{d+1}=(-1)^{d+1}(d+1) ! \bigwedge_{k=2}^{m}\left(\omega_{1 k} \wedge \omega_{k 1}\right), \quad\left(d_{\wedge} \omega_{11}\right)^{d+2}=0 ; \\
& \left(d_{\wedge} \omega_{22}\right)^{d+1}=(-1)^{d+1}(d+1) ! \omega_{21} \wedge \omega_{12} \wedge\left(\bigwedge_{k=3}^{m}\left(\omega_{2 k} \wedge \omega_{k 2}\right)\right), \quad\left(d_{\wedge} \omega_{22}\right)^{d+2}=0 ; \\
& \left(d_{\wedge} \omega_{11}\right)^{d+1} \wedge\left(d_{\wedge} \omega_{22}\right)^{d}=-\left(d_{\wedge} \omega_{11}\right)^{d} \wedge\left(d_{\wedge} \omega_{22}\right)^{d+1} \\
& =-(d+1) ! d !\left(\bigwedge_{k=2}^{m}\left(\omega_{1 k} \wedge \omega_{k 1}\right)\right) \wedge\left(\bigwedge_{k=3}^{m}\left(\omega_{2 k} \wedge \omega_{k 2}\right)\right) ; \\
& \left(d_{\wedge} \tau_{1}^{\prime}\right)^{j} \wedge\left(d_{\wedge} \tau_{2}^{\prime}\right)^{q_{2}-j}=-(d+2)^{j}(d+1)^{d}(d-j)\left(\begin{array}{c}
q_{2}-j \\
d
\end{array}\right) \\
& \quad \times\left(d_{\wedge} \omega_{11}\right)^{d+1} \wedge\left(d_{\wedge} \omega_{22}\right)^{d}, \quad 0 \leqq j \leqq q_{1}=d+1 .
\end{aligned}
$$

Par suite, on a donc 


$$
\begin{aligned}
& {\left[\tau_{2} \wedge\left(d \tau_{1}\right)^{j} \wedge\left(d \tau_{2}\right)^{q_{2}-j}\right]=0 \in H_{D R}^{2 q_{2}+1}(M), \quad 0 \leqq j \leqq q_{1}} \\
& {\left[\tau_{1} \wedge \tau_{2} \wedge\left(d \tau_{1}\right)^{d} \wedge\left(d \tau_{2}\right)^{q_{1}}\right]=0 \in H_{D R}^{2 q_{2}+2}(M)}
\end{aligned}
$$

En particulier, la classe de Godbillon-Vey du feuilletage $F_{2}$ est nulle.

Pour démontrer que les autres classes de Godbillon-Vey du sousfeuilletage $\left(F_{1}, F_{2}\right)$ ne sont pas nulles, nous procédons comme suit. Puisque $\tau_{1}^{\prime} \wedge\left(d_{\wedge} \tau_{1}^{\prime}\right)^{d+1}=(d+2)^{d+2} \omega_{11} \wedge\left(d_{\wedge} \omega_{11}\right)^{d+1}$ est un élément non nul dans $\left(\wedge^{p} \bar{g}^{*}\right)_{s L(d+1)}$, avec $p=\operatorname{dim} \bar{g} / s l(d+1)=2 q_{1}+1$, il en résulte que la classe $\left[\tau_{1}^{\prime} \wedge\left(d_{\wedge} \tau_{1}^{\prime}\right)^{d+1}\right] \in H(\bar{g}, H)$ est non nulle. De même, pour chaque $j=0,1, \cdots$, $q_{1}, j \neq d$, l'élément

$$
\begin{aligned}
& \tau_{1}^{\prime} \wedge \tau_{2}^{\prime} \wedge\left(d_{\wedge} \tau_{1}^{\prime}\right)^{j} \wedge\left(d_{\wedge} \tau_{2}^{\prime}\right)^{q_{2}-j}=-(d+2)^{j+1}(d+1)^{d+1}(d-j)\left[\begin{array}{c}
q_{2}-j \\
d
\end{array}\right] \\
& \omega_{11} \wedge \omega_{22} \wedge\left(d_{\wedge} \omega_{11}\right)^{d+1} \wedge\left(d_{\wedge} \omega_{22}\right)^{d}
\end{aligned}
$$

n'est pas nul dans $\left(\wedge^{q} \bar{g}^{*}\right)_{s L(d)}$, avec $q=\operatorname{dim} \bar{g} / s l(d)=2 q_{2}+2$. Par conséquent, la classe $\left[\tau_{1}^{\prime} \wedge \tau_{2}^{\prime} \wedge\left(d_{\wedge} \tau_{1}^{\prime}\right)^{j} \wedge\left(d_{\wedge} \tau_{2}^{\prime}\right)^{q_{2}-j}\right] \in H(\bar{g}, H)$ est non nulle pour 0 $\leqq j \leqq q_{1}$ et $j \neq d$. Comme l'algèbre $H(\bar{g}, H)$ satisfait à la dualité de Poincaré (par rapport à un élément non nul dans $\left(\wedge^{n} \bar{g}^{*}\right)_{H}$, avec $n=\operatorname{dim} \bar{g} / h=$ $\operatorname{dim} M$ ), et comme la variété $M$ est compacte et orientable, on a obtenu le résultat suivant.

Théorème 6.1. Pour le sous-feuilletage localement homogène $\left(F_{1}, F_{2}\right)=$ $\left(F_{S L(d+2,1)_{0}}, F_{S L(d+2,2)_{0}}\right)$ de codimension $\left(q_{1}, q_{2}\right)=(d+1,2 d+1)$ sur $M=$ $\Gamma \backslash S L(d+2) / S O(d)$, les classes de Godbillon-Vey $\left[\tau_{1} \wedge\left(d \tau_{1}\right)^{q_{1}}\right] \in H_{D R}^{2 q_{1}+1}(M)$ et $\left[\tau_{1} \wedge \tau_{2} \wedge\left(d \tau_{1}\right)^{j} \wedge\left(d \tau_{2}\right)^{q_{2}-j}\right] \in H_{D R}^{2 q_{2}+2}(M), \quad 0 \leqq j \leqq q_{1}, \quad j \neq d$, sont non nulles. Ainsi, d'après le Corollaire 4.5, les fibrés $Q_{2}$ et $Q_{1} \oplus Q_{0}$ ne sont pas isomorphes comme fibrés vectoriels $F_{2}$-feuilletés.

Ceci montre que le fibré principal $\left(F_{1}, F_{2}\right)$-feuilleté $P=L\left(Q_{1}\right)+L\left(Q_{0}\right)$ des repères transverses à $\left(F_{1}, F_{2}\right)$ n'admet pas une connexion basique somme et que la structure $\left(F_{1}, F_{2}\right)$-feuilletée de $P$ n'est pas induite par une structure $\left(F_{1}, F_{2}\right)$-feuilletée de une $G_{1}{ }^{\prime} \times G_{2}{ }^{\prime}$-réduction $P^{\prime}=P_{1}{ }^{\prime}+P_{2}^{\prime}$ de $P$, où $G_{1}{ }^{\prime} \times G_{2}{ }^{\prime}$ désigne l'un des groupes suivants :

$$
S L\left(q_{1}\right) \times S L(d), \quad G L\left(q_{1}\right) \times S L(d), \quad S L\left(q_{1}\right) \times G L(d) .
$$

Remarque. Si $d=1$, alors, pour chaque $j=0,2$, on vérifie que $\tau_{1} \wedge \tau_{2} \wedge\left(d \tau_{1}\right)^{j} \wedge\left(d \tau_{2}\right)^{3-j}$ est une forme volume de la variété compacte $M=$ $\Gamma \backslash S L(3)$. Par suite, on peut considérer ce cas comme une généralisation de l'exemple de Roussarie [5]. 
Exemple 2. Pour $d \geqq 1$, considérons les groupes de Lie $\bar{G}=S L(d+2)$, $G_{1}=S L(d+2,1), \quad G_{2}=S L(d+2,2), \quad H=O(d)$ (ou $\left.O(1) \times O(d)\right)$ et $\Gamma \subset$ $S L(d+2)$ un sous-groupe discret, uniforme et sans torsion. Alors le fibré normal $Q_{1} \oplus Q_{0}$ du sous-feuilletage localement homogène $\left(F_{1}, F_{2}\right)=\left(F_{G_{1}}\right.$, $\left.F_{G_{2}}\right)$ de codimension $\left(q_{1}, q_{2}\right)=(d+1,2 d+1)$ sur la variété compacte connexe $M=\Gamma \backslash \bar{G} / H$ n'est pas nécessairement orientable.

Soit maintenant $\rho$ la représentation canonique de $H$ dans $\wedge \bar{g}^{*}$. Avec les notations de l'Exemple 1 , on obtient alors $\rho(\bar{A}) \omega_{11}=\omega_{11}, \quad \rho(\bar{A}) \omega_{22}=$ $\omega_{22}, \rho(\bar{A}) \gamma_{1}^{\prime}=\lambda^{d}$ dét $A^{d} \cdot \gamma_{1}^{\prime}$ et $\rho(\bar{A}) \gamma_{2}^{\prime}=\lambda^{d} \operatorname{dét} A \cdot \gamma_{2}^{\prime}$ pour $\bar{A}=(\lambda, A) \in H$, où $\lambda=1$ si $H=O(d)$. Par conséquent, pour chaque $i=1,2$, lélément $\tau_{i}^{\prime} \in$ $\left(\wedge \bar{g}^{*}\right)_{H}$ induit une 1-forme $\tau_{i}$ sur $M$. De même, $\gamma_{1}^{\prime}$ (resp. $\gamma_{2}^{\prime}$ ) détermine un produit intérieur euclidien $H$-invariant sur $\wedge^{d+1}\left(\bar{g} / g_{1}\right) *$ (resp. sur $\wedge^{d}\left(g_{1} /\right.$ $\left.g_{2}\right)^{*}$ ) tel que $\gamma_{1}^{\prime}$ (resp. $\gamma_{2}^{\prime}$ ) soit de norme 1. Ainsi, pour appliquer le Théorème 5.2, on peut utiliser les métriques riemanniennes sur $\wedge^{d+1} Q_{1}{ }^{*} \cong$ $\Gamma \backslash \bar{G} \times{ }_{H} \wedge^{d+1}\left(\bar{g} / g_{1}\right)^{*}$ et $\wedge^{d} Q_{0}{ }^{*} \cong \Gamma \backslash \bar{G} \times{ }_{H} \wedge^{d}\left(g_{1} / g_{2}\right)^{*}$ induites par les produits scalaires précédents. D'autre part, on obtient un recouvrement ouvert $\{U\}$ de $M$ et des repères mobiles normés $\gamma_{1}{ }^{U}$ et $\gamma_{2}{ }^{U}$ sur $U$ pour $\wedge^{d+1} Q_{1}{ }^{*}$ et $\wedge^{d} Q_{0}{ }^{*}$ (par rapport à ces métriques) tels que

$$
d \gamma_{1}{ }^{U}=\tau_{1} \wedge \gamma_{1}{ }^{U} \quad \text { et } \quad d \gamma_{2}{ }^{U}-\tau_{2} \wedge \gamma_{2}{ }^{U} \in F^{1} \Omega(U) .
$$

Comme l'homomorphisme canonique $H(\bar{g}, H) \longrightarrow H(\bar{g}, S O(d))$ est injectif, il en résulte que les classes de Godbillon-Vey de $\left(F_{1}, F_{2}\right)$ sont données par l'Exemple 1 et le Théorème 6.1. En particulier, $Q_{2}$ et $Q_{1} \oplus Q_{0}$ ne sont pas isomorphes comme fibrés vectoriels $F_{2}$-feuilletés.

Il est clair que l'application canonique

$$
f: M^{\prime}=\Gamma \backslash S L(d+2) / S O(d) \longrightarrow M=\Gamma \backslash S L(d+2) / H
$$

est une application de variétés sous-feuilletées. Par suite, l'homomorphisme $f^{*}: H_{D R}(M) \longrightarrow H_{D R}\left(M^{\prime}\right)$ transforme chaque classe de Godbillon-Vey de $\left(F_{S L(d+2,1)}, F_{S L(d+2,2)}\right)$ en la classe correspondante de Godbillon-Vey de $\left(F_{S L(d+2,1)_{0}}, F_{S L(d+2,2)_{0}}\right)$.

Remarques. 1) Si, dans les Exemples 1 et 2, on remplace les groupes $G_{i}, i=1,2$, par les groupes $G_{i}{ }^{t}$ des matrices transposées de celles de $G_{i}$, on vérifie que les 1 -formes $\tau_{i}^{t}, i=1,2$, sur $M=\Gamma \backslash \bar{G} / H$, obtenues pour les nouveaux sous-feuilletages, sont données par $\tau_{i}{ }^{t}=-\tau_{i}, i=1,2$, où $\tau_{1}$ et $\tau_{2}$ sont les 1 -formes sur $M$ construites dans les Exemples 1 et 2 . Il s'ensuit que 
$\left[\tau_{1}^{t} \wedge \tau_{2}^{t} \wedge\left(d \tau_{1}^{t}\right)^{j} \wedge\left(d \tau_{2}{ }^{t}\right)^{q_{2}-j}\right]=-\left[\tau_{1} \wedge \tau_{2} \wedge\left(d \tau_{1}\right)^{j} \wedge\left(d \tau_{2}\right)^{q_{2}-j}\right] \neq 0 \in H_{D R}^{2 q_{2}+2}(M)$, $0 \leqq j \leqq q_{1}, \quad j \neq d$.

Par conséquent, il existe au moins deux sous-feuilletages de codimension $\left(q_{1}, q_{2}\right)=(d+1,2 d+1)$ sur $M$, qui ne sont pas intégrablement homotopes.

2) Dans une publication ultérieure, en utilisant l'homomorphisme caractéristique d'un sous-feuilletage, nous montrerons que, si $d$ est impair, les classes de Godbillon-Vey non nulles de degré $2 q_{2}+2$ des sousfeuilletages considérés dans les exemples précédents sont des invariants (non nuls) de ces sous-feuilletages, qui n'appartiennent pas à la sousalgèbre de $H_{D R}(M)$ engendrée par les classes caractéristiques des feuilletages de la paire. De même, la non trivialité de ces classes implique que les algèbres de Lie $s l(d+2, i), i=1,2$, ne sont pas réductives. De plus, on vérifie que, si $d$ est pair, aucun feuilletage intégrablement homotope à $F_{1}$ ne peut se prolonger en un feuilletage de codimension $q$ pour $0<q<q_{1}=d+1$.

3) Pour un sous-feuilletage localement homogène $\left(F_{1}, F_{2}\right)=\left(F_{G_{1}}, F_{G_{2}}\right)$ de codimension $\left(q_{1}, q_{2}\right)$ sur $M=\Gamma \backslash \bar{G} / H$, on peut facilement démontrer que $Q_{2}$ et $Q_{1} \oplus Q_{0}$ sont isomorphes comme fibrés vectoriels $F_{2}$-feuilletés, pourvu que $g_{2}$ soit une sous-algèbre réductive dans $\bar{g}$ et que $G_{2}$ ait un nombre fini de composantes connexes. C'est par exemple le cas si $\bar{G}=S L(q+2), G_{1}=$ $G L(q+1), G_{2}=G L(1) \times G L(q), H=O(q)$ et $\Gamma \subset S L(q+2)$ un sous-groupe discret, uniforme et sans torsion.

De la même manière, s'il existe un sous-groupe de Lie $G^{\prime} \subset \bar{G}$ tel que $G_{2}$ $=G_{1} \cap G^{\prime}$ et $\operatorname{dim} \bar{g} / g^{\prime}=d=q_{2}-q_{1}\left(g^{\prime}\right.$ étant l'algèbre de Lie de $\left.G^{\prime}\right)$, alors $F_{2}$ $=F_{1} \cap F^{\prime}$, où $F^{\prime}$ désigne le feuilletage localement homogène $F_{G^{\prime}}$, de codimension $d$ sur $M$. Un exemple de ce cas s'obtient en prenant $\bar{G}=$ $S L(d+1), G_{1}=S L(d+1,1), G^{\prime}=S L(d+1,1)^{t}, G_{2}=G L(d), H=O(d)$ et $\Gamma \subset$ $S L(d+1)$ un sous-groupe discret, uniforme et sans torsion. De même, on peut prendre $\bar{G}=U(q+1), G_{1}=S U(q+1), G^{\prime}=U(1) \times U(q), G_{2}=U(q), H=$ $O(q)$ et $\Gamma=\{e\}$.

4) Avec les notations de la Proposition 2.1, on démontre sans difficulté que l'homomorphisme caractêristique $\Delta_{*}: H\left(W(g, H)_{I^{\prime}}\right) \longrightarrow H_{D R}(M)$ de chacun des sous-feuilletages localement homogènes suivants (à connexion basique somme) dépend du choix de la connexion basique somme ( $I^{\prime}$ étant l'idéal correspondant à la paire $\left.\left(\left[q_{1} / 2\right],\left[q_{2} / 2\right]\right)\right)$ :

i) $\left(F_{1}, F_{2}\right)=\left(F_{U(2)}, F_{U(1)}\right),\left(q_{1}, q_{2}\right)=(2,5)$ et $M=S O(4)$.

ii) $\left(F_{1}, F_{2}\right)=\left(F_{U(q)}, F_{S U(q)}\right),\left(q_{1}, q_{2}\right)=(2 q+1,2 q+2)$ et $M=U(q+1)$. 
iii) $\left(F_{1}, F_{2}\right)=\left(F_{S O(2 n) \times U(q+1)}, F_{S O(2 n) \times U(q)}\right),\left(q_{1}, q_{2}\right)=(2 n, 2 n+2 q+1)$ et $M$ $=S O(2 n+1) \times U(q+1)$.

iv) $\left(F_{1}, F_{2}\right)=\left(F_{G L(1)}, 0\right),\left(q_{1}, q_{2}\right)=(2,3)$ et $M=\Gamma \backslash S L(2)$, où $\Gamma \subset S L(2)$ est un sous-groupe discret et uniforme.

Dans le cas d'un feuilletage localement homogène à connexion basique dont l'homomorphisme caractéristique $\Delta_{*}: H\left(W(g, H)_{[q / 2]}\right) \longrightarrow H_{D R}(M)$ dépend du choix de la connexion basique ( $q$ étant la codimension du feuilletage $F$ sur $M$ ), nous donnons les exemples suivants :

i) $F=F_{U\left(q^{\prime}\right)}\left(\right.$ resp. $\left.F=F_{S U\left(q^{\prime}\right)}\right), q=2 q^{\prime}+1$ et $M=U\left(q^{\prime}+1\right)($ resp. $M=$ $\left.S U\left(q^{\prime}+1\right)\right)$.

ii) $F=F_{S o(2 n-1)}, q=4 n-1$ et $M=S O(2 n+1)$.

iii) $F=F_{S L(1)}=0, q=3$ et $M=\Gamma \backslash S L(2)$, où $\Gamma \subset S L(2)$ est un sousgroupe discret et uniforme.

Ainsi, en vertu du Corollaire 2.2, on a obtenu le résultat suivant.

Proposition 6.2. L'ensemble (supposé non vide) des connexions basiques à un feuilletage de codimension $q$ est $J(>[(q+1) / 2])$-connexe (au sens de Lehmann[9]), mais cet ensemble n'est généralement pas $J(>[q / 2])$-connexe si $q$ est impair.

\section{References}

[1] Bott, R., Lectures on characteristic classes and foliations, Lecture Notes in Math., 279 (1972), Springer, Berlin, 1-94.

[2] Carballés, J. M., Characteristic homomorphism for $\left(F_{1}, F_{2}\right)$-foliated bundles over subfoliated manifolds, Ann. Inst. Fourier, Grenoble, 33, 3 (1984), 219-245.

[ 3 ] Cordero, L. A. et Masa, X., Characteristic classes of subfoliations, Ann. Inst. Fourier, Grenoble, 31, 2 (1981), 61-86.

[4] Feigin, B. L., Characteristic classes of flags of foliations, Funct. Anal. and its Appl., 9 (1975), 312-317.

[ 5 ] Godbillon, C. et Vey, J., Un invariant des feuilletages de codimension 1, C. R. Acad, Sci. Paris, 273 (1971), 92-95.

[6] Greub, W.. Halperin, S. et Vanstone, R., Connections, curvature and cohomology, Academic Press, New York, Vol. I (1972), Vol. II (1973), Vol. III (1976).

[ 7 ] Heitsch, J. L., Deformations of secondary characteristic classes, Topology, 12 (1973), 381 388.

[ 8 ] Kamber, F. W. et Tondeur, Ph., Foliated bundles and characteristic classes, Lecture Notes in Math., 493 (1975), Springer, Berlin.

[ 9 ] Lehmann, D., Classes caractéristiques exotiques et J-connexité des espaces de connexions, Ann. Inst. Fourier, Grenoble, 24, 3 (1974), 267-306.

[10] Masa, X., Characteristic classes of subfoliations II, preprint.

[11] Molino, P., Propriétés cohomologiques et propriétés topologiques des feuilletages à 
connexion transverse projetable, Topology, 12 (1973), 317-325.

[12] Moussu, R., Sur les classes exotiques des feuilletages, Lecture Notes in Math., 392 (1974), Springer, Berlin, 37-42.

[13] Vaisman, I., Cohomology and differential forms, Marcel Dekker, Inc., New York (1973).

[14] Yamato, K., Examples of foliations with non-trivial exotic characteristic classes, Osaka J. Math., 12 (1975), 401-417. 